EUROPEAN LABORATORY FOR PARTICLE PHYSICS

CERN-EP/98-136

18th August 1998

\title{
Search for Chargino and Neutralino Production at $\sqrt{s}=181-184 \mathrm{GeV}$ at LEP
}

\author{
The OPAL Collaboration
}

\begin{abstract}
A search for charginos and neutralinos, predicted by supersymmetric theories, has been performed using a data sample of $57 \mathrm{pb}^{-1}$ at centre-of-mass energies of $181-184 \mathrm{GeV}$ taken with the OPAL detector at LEP. No evidence for chargino or neutralino production has been found. Upper limits on chargino and neutralino pair production $\left(\tilde{\chi}_{1}^{+} \tilde{\chi}_{1}^{-}, \tilde{\chi}_{1}^{0} \tilde{\chi}_{2}^{0}\right)$ crosssections are obtained as a function of the chargino mass $\left(m_{\tilde{\chi}_{1}^{ \pm}}\right)$, the lightest neutralino mass $\left(m_{\tilde{\chi}_{1}^{0}}\right)$ and the second lightest neutralino mass $\left(m_{\tilde{\chi}_{2}^{0}}\right)$. For large chargino masses the limits have been improved with respect to the previous analyses at lower centre-ofmass energies. Exclusion regions at 95\% confidence level (C.L.) of parameters of the Constrained Minimal Supersymmetric Standard Model are determined for the case of a large universal scalar mass, $m_{0}$, implying heavy scalar fermions, and for the case of a small $m_{0}$ resulting in light scalar fermions and giving the worst-case limits. Within this framework and for $m_{\tilde{\chi}_{1}^{ \pm}}-m_{\tilde{\chi}_{1}^{0}} \geq 5 \mathrm{GeV}$ the $95 \%$ C.L. lower limits on $m_{\tilde{\chi}_{1}^{ \pm}}$for $m_{0}=$ $500 \mathrm{GeV}$ are 90.0 and $90.2 \mathrm{GeV}$ for $\tan \beta=1.5$ and 35 respectively. These limits for all $m_{0}$ (the worst-case) are 69.1 and $65.2 \mathrm{GeV}$ for $\tan \beta=1.5$ and 35 respectively. Exclusion regions are also presented for neutralino masses, including an absolute lower limit at 95\% C.L. for the mass of the lightest neutralino of $30.1 \mathrm{GeV}$ for $m_{0}=500 \mathrm{GeV}(24.2 \mathrm{GeV}$ for all $m_{0}$ ), with implications for experimental searches for the lightest neutralino as a dark matter candidate.
\end{abstract}

Submitted to Eur. Phys. Journal C 
G. Abbiendi ${ }^{2}$, K. Ackerstaff ${ }^{8}$, G. Alexander ${ }^{23}$, J. Allison ${ }^{16}$, N. Altekamp ${ }^{5}$, K.J. Anderson ${ }^{9}$, S. Anderson ${ }^{12}$, S. Arcelli ${ }^{17}$, S. Asai ${ }^{24}$, S.F. Ashby ${ }^{1}$, D. Axen ${ }^{29}$, G. Azuelos ${ }^{18, a}$, A.H. Ball ${ }^{17}$,

E. Barberio ${ }^{8}$, R.J. Barlow ${ }^{16}$, R. Bartoldus ${ }^{3}$, J.R. Batley ${ }^{5}$, S. Baumann ${ }^{3}$, J. Bechtluft ${ }^{14}$, T. Behnke ${ }^{27}$, K.W.Bell ${ }^{20}$, G. Bella ${ }^{23}$, A. Bellerive ${ }^{9}$, S. Bentvelsen ${ }^{8}$, S. Bethke ${ }^{14}$, S. Betts ${ }^{15}$, O. Biebel ${ }^{14}$, A. Biguzzi ${ }^{5}$, S.D. Bird ${ }^{16}$, V. Blobel ${ }^{27}$, I.J.Bloodworth ${ }^{1}$, M. Bobinski ${ }^{10}$, P. Bock ${ }^{11}$, J. Böhme ${ }^{14}$, D. Bonacorsi ${ }^{2}$, M. Boutemeur ${ }^{34}$, S. Braibant ${ }^{8}$, P. Bright-Thomas ${ }^{1}$, L. Brigliadori ${ }^{2}$, R.M. Brown ${ }^{20}$, H.J. Burckhart ${ }^{8}$, C. Burgard ${ }^{8}$, R. Bürgin ${ }^{10}$, P. Capiluppi ${ }^{2}$, R.K. Carnegie ${ }^{6}$, A.A. Carter ${ }^{13}$, J.R. Carter ${ }^{5}$, C.Y. Chang ${ }^{17}$, D.G. Charlton ${ }^{1, b}$, D. Chrisman ${ }^{4}$, C. Ciocca ${ }^{2}$, P.E.L. Clarke ${ }^{15}$, E. Clay ${ }^{15}$, I. Cohen ${ }^{23}$, J.E. Conboy ${ }^{15}$, O.C. Cooke ${ }^{8}$, C. Couyoumtzelis ${ }^{13}$, R.L. Coxe ${ }^{9}$, M.Cuffiani ${ }^{2}$, S. Dado ${ }^{22}$, G.M. Dallavalle ${ }^{2}$, R. Davis ${ }^{30}$, S. De Jong ${ }^{12}$, L.A. del Pozo ${ }^{4}$, A. de Roeck ${ }^{8}$, K. Desch ${ }^{8}$, B. Dienes ${ }^{33, d}$, M.S. Dixit ${ }^{7}$, J.Dubbert ${ }^{34}$, E. Duchovni ${ }^{26}$, G. Duckeck ${ }^{34}$,

I.P. Duerdoth ${ }^{16}$, D. Eatough ${ }^{16}$, P.G. Estabrooks ${ }^{6}$, E. Etzion ${ }^{23}$, H.G. Evans ${ }^{9}$, F. Fabbri ${ }^{2}$, M. Fanti ${ }^{2}$, A.A. Faust ${ }^{30}$, F. Fiedler ${ }^{27}$, M. Fierro ${ }^{2}$, I. Fleck ${ }^{8}$, R. Folman ${ }^{26}$, A. Fürtjes ${ }^{8}$, D.I. Futyan ${ }^{16}$, P. Gagnon ${ }^{7}$, J.W. Gary ${ }^{4}$, J. Gascon ${ }^{18}$, S.M. Gascon-Shotkin ${ }^{17}$, G. Gaycken ${ }^{27}$, C. Geich-Gimbel ${ }^{3}$, G. Giacomelli², P. Giacomelli², V. Gibson ${ }^{5}$, W.R. Gibson ${ }^{13}$,

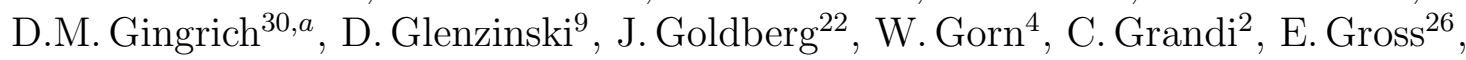
J. Grunhaus ${ }^{23}$, M. Gruwé27, G.G. Hanson ${ }^{12}$, M. Hansroul ${ }^{8}$, M. Hapke ${ }^{13}$, K. Harder ${ }^{27}$, C.K. Hargrove ${ }^{7}$, C. Hartmann ${ }^{3}$, M. Hauschild ${ }^{8}$, C.M. Hawkes ${ }^{5}$, R. Hawkings ${ }^{27}$, R.J.Hemingway ${ }^{6}$, M. Herndon ${ }^{17}$, G. Herten ${ }^{10}$, R.D. Heuer ${ }^{8}$, M.D. Hildreth ${ }^{8}$, J.C. Hill ${ }^{5}$, S.J.Hillier ${ }^{1}$, P.R. Hobson ${ }^{25}$, A. Hocker ${ }^{9}$, R.J. Homer ${ }^{1}$, A.K. Honma ${ }^{28, a}$, D. Horváth ${ }^{32, c}$, K.R. Hossain ${ }^{30}$, R. Howard ${ }^{29}$, P. Hüntemeyer ${ }^{27}$, P. Igo-Kemenes ${ }^{11}$, D.C. Imrie ${ }^{25}$, K. Ishii ${ }^{24}$, F.R. Jacob ${ }^{20}$, A. Jawahery ${ }^{17}$, H. Jeremie ${ }^{18}$, M. Jimack ${ }^{1}$, C.R. Jones ${ }^{5}$, P. Jovanovic ${ }^{1}$, T.R. Junk ${ }^{6}$, D. Karlen ${ }^{6}$, V. Kartvelishvili ${ }^{16}$, K. Kawagoe ${ }^{24}$, T. Kawamoto ${ }^{24}$, P.I. Kayal ${ }^{30}$, R.K. Keeler ${ }^{28}$,

R.G.Kellogg ${ }^{17}$, B.W. Kennedy ${ }^{20}$, A. Klier ${ }^{26}$, S. Kluth ${ }^{8}$, T. Kobayashi ${ }^{24}$, M. Kobel ${ }^{3, e}$, D.S. Koetke ${ }^{6}$, T.P. Kokott ${ }^{3}$, M. Kolrep ${ }^{10}$, S. Komamiya ${ }^{24}$, R.V.Kowalewski ${ }^{28}$, T. Kress ${ }^{11}$, P. Krieger ${ }^{6}$, J. von Krogh ${ }^{11}$, T. Kuhl ${ }^{3}$, P. Kyberd ${ }^{13}$, G.D. Lafferty ${ }^{16}$, D. Lanske ${ }^{14}$, J. Lauber ${ }^{15}$, S.R. Lautenschlager ${ }^{31}$, I. Lawson ${ }^{28}$, J.G. Layter ${ }^{4}$, D. Lazic ${ }^{22}$, A.M. Lee ${ }^{31}$, D. Lellouch ${ }^{26}$, J. Letts ${ }^{12}$, L. Levinson ${ }^{26}$, R. Liebisch ${ }^{11}$, B. List ${ }^{8}$, C. Littlewood ${ }^{5}$, A.W. Lloyd ${ }^{1}$, S.L. Lloyd ${ }^{13}$, F.K. Loebinger ${ }^{16}$, G.D. Long ${ }^{28}$, M.J. Losty ${ }^{7}$, J. Ludwig ${ }^{10}$, D. Liu ${ }^{12}$, A. Macchiolo ${ }^{2}$, A. Macpherson ${ }^{30}$, W. Mader ${ }^{3}$, M. Mannelli ${ }^{8}$, S. Marcellini' ${ }^{2}$, C. Markopoulos ${ }^{13}$, A.J. Martin ${ }^{13}$, J.P. Martin ${ }^{18}$, G. Martinez ${ }^{17}$, T. Mashimo ${ }^{24}$, P. Mättig ${ }^{26}$, W.J. McDonald ${ }^{30}$, J. McKenna ${ }^{29}$, E.A. Mckigney ${ }^{15}$, T.J. McMahon ${ }^{1}$, R.A. McPherson ${ }^{28}$, F. Meijers ${ }^{8}$, S. Menke ${ }^{3}$, F.S. Merritt ${ }^{9}$, H. Mes ${ }^{7}$, J. Meyer ${ }^{27}$, A. Michelini' ${ }^{2}$, S. Mihara ${ }^{24}$, G. Mikenberg ${ }^{26}$, D.J. Miller ${ }^{15}$, R. Mir ${ }^{26}$, W. Mohr ${ }^{10}$, A. Montanari ${ }^{2}$, T. Mori2 ${ }^{24}$, K. Nagai ${ }^{8}$, I. Nakamura ${ }^{24}$, H.A. Neal ${ }^{12}$, B. Nellen ${ }^{3}$, R. Nisius ${ }^{8}$, S.W. O'Neale ${ }^{1}$, F.G. Oakham ${ }^{7}$, F. Odorici ${ }^{2}$, H.O. Ogren ${ }^{12}$, M.J. Oreglia' ${ }^{9}$, S. Orito ${ }^{24}$, J.Pálinkás $33, d$, G. Pásztor ${ }^{32}$, J.R.Pater ${ }^{16}$, G.N. Patrick ${ }^{20}$, J. Patt ${ }^{10}$, R. Perez-Ochoa ${ }^{8}$, S. Petzold ${ }^{27}$, P. Pfeifenschneider ${ }^{14}$, J.E. Pilcher ${ }^{9}$, J. Pinfold ${ }^{30}$, D.E. Plane ${ }^{8}$, P. Poffenberger ${ }^{28}$, J. Polok ${ }^{8}$, M. Przybycień ${ }^{8}$, C. Rembser ${ }^{8}$, H. Rick ${ }^{8}$, S. Robertson ${ }^{28}$, S.A. Robins ${ }^{22}$, N. Rodning ${ }^{30}$, J.M. Roney ${ }^{28}$, K. Roscoe ${ }^{16}$, A.M. Rossi ${ }^{2}$, Y. Rozen ${ }^{22}$, K. Runge ${ }^{10}$, O. Runolfsson ${ }^{8}$, D.R. Rust ${ }^{12}$, K. Sachs ${ }^{10}$, T. Saeki ${ }^{24}$, O. Sahr ${ }^{34}$, W.M. Sang ${ }^{25}$, E.K.G. Sarkisyan ${ }^{23}$, C. Sbarra ${ }^{29}$, A.D. Schaile ${ }^{34}$,

O. Schaile ${ }^{34}$, F. Scharf ${ }^{3}$, P. Scharff-Hansen ${ }^{8}$, J. Schieck ${ }^{11}$, B. Schmitt ${ }^{8}$, S. Schmitt ${ }^{11}$, A. Schöning ${ }^{8}$, M. Schröder ${ }^{8}$, M. Schumacher ${ }^{3}$, C. Schwick ${ }^{8}$, W.G. Scott ${ }^{20}$, R. Seuster ${ }^{14}$, T.G. Shears ${ }^{8}$, B.C. Shen ${ }^{4}$, C.H. Shepherd-Themistocleous ${ }^{8}$, P. Sherwood ${ }^{15}$, G.P. Siroli ${ }^{2}$, A. Sittler ${ }^{27}$, A. Skuja ${ }^{17}$, A.M. Smith ${ }^{8}$, G.A. Snow ${ }^{17}$, R. Sobie ${ }^{28}$, S. Söldner-Rembold ${ }^{10}$, M. Sproston ${ }^{20}$, A. Stahl ${ }^{3}$, K. Stephens ${ }^{16}$, J.Steuerer ${ }^{27}$, K. Stoll ${ }^{10}$, D. Strom ${ }^{19}$, R. Ströhmer ${ }^{34}$, B. Surrow ${ }^{8}$, S.D. Talbot ${ }^{1}$, S. Tanaka ${ }^{24}$, P. Taras ${ }^{18}$, S. Tarem ${ }^{22}$, R. Teuscher ${ }^{8}$, M. Thiergen ${ }^{10}$, M.A. Thomson ${ }^{8}$, E. von Törne ${ }^{3}$, E. Torrence ${ }^{8}$, S. Towers ${ }^{6}$, I. Trigger ${ }^{18}$, Z. Trócsányi ${ }^{33}$, 
E. Tsur ${ }^{23}$, A.S. Turcot ${ }^{9}$, M.F. Turner-Watson ${ }^{8}$, R. Van Kooten ${ }^{12}$, P. Vannerem ${ }^{10}$,

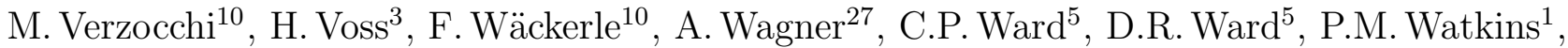
A.T. Watson ${ }^{1}$, N.K. Watson ${ }^{1}$, P.S. Wells ${ }^{8}$, N. Wermes ${ }^{3}$, J.S. White ${ }^{6}$, G.W.Wilson ${ }^{16}$, J.A. Wilson ${ }^{1}$, T.R. Wyatt $^{16}$, S. Yamashita ${ }^{24}$, G. Yekutieli ${ }^{26}$, V. Zacek ${ }^{18}$, D. Zer-Zion ${ }^{8}$

${ }^{1}$ School of Physics and Astronomy, University of Birmingham, Birmingham B15 2TT, UK

${ }^{2}$ Dipartimento di Fisica dell' Università di Bologna and INFN, I-40126 Bologna, Italy

${ }^{3}$ Physikalisches Institut, Universität Bonn, D-53115 Bonn, Germany

${ }^{4}$ Department of Physics, University of California, Riverside CA 92521, USA

${ }^{5}$ Cavendish Laboratory, Cambridge CB3 0HE, UK

${ }^{6}$ Ottawa-Carleton Institute for Physics, Department of Physics, Carleton University, Ottawa, Ontario K1S 5B6, Canada

${ }^{7}$ Centre for Research in Particle Physics, Carleton University, Ottawa, Ontario K1S 5B6, Canada

${ }^{8}$ CERN, European Organisation for Particle Physics, CH-1211 Geneva 23, Switzerland

${ }^{9}$ Enrico Fermi Institute and Department of Physics, University of Chicago, Chicago IL 60637, USA

${ }^{10}$ Fakultät für Physik, Albert Ludwigs Universität, D-79104 Freiburg, Germany

${ }^{11}$ Physikalisches Institut, Universität Heidelberg, D-69120 Heidelberg, Germany

${ }^{12}$ Indiana University, Department of Physics, Swain Hall West 117, Bloomington IN 47405, USA

${ }^{13}$ Queen Mary and Westfield College, University of London, London E1 4NS, UK

${ }^{14}$ Technische Hochschule Aachen, III Physikalisches Institut, Sommerfeldstrasse 26-28, D-52056 Aachen, Germany

${ }^{15}$ University College London, London WC1E 6BT, UK

${ }^{16}$ Department of Physics, Schuster Laboratory, The University, Manchester M13 9PL, UK

${ }^{17}$ Department of Physics, University of Maryland, College Park, MD 20742, USA

${ }^{18}$ Laboratoire de Physique Nucléaire, Université de Montréal, Montréal, Quebec H3C 3J7, Canada

${ }^{19}$ University of Oregon, Department of Physics, Eugene OR 97403, USA

${ }^{20}$ CLRC Rutherford Appleton Laboratory, Chilton, Didcot, Oxfordshire OX11 0QX, UK

${ }^{22}$ Department of Physics, Technion-Israel Institute of Technology, Haifa 32000, Israel

${ }^{23}$ Department of Physics and Astronomy, Tel Aviv University, Tel Aviv 69978, Israel

${ }^{24}$ International Centre for Elementary Particle Physics and Department of Physics, University of Tokyo, Tokyo 113-0033, and Kobe University, Kobe 657-8501, Japan

${ }^{25}$ Institute of Physical and Environmental Sciences, Brunel University, Uxbridge, Middlesex UB8 3PH, UK

${ }^{26}$ Particle Physics Department, Weizmann Institute of Science, Rehovot 76100, Israel

${ }^{27}$ Universität Hamburg/DESY, II Institut für Experimental Physik, Notkestrasse 85, D-22607 Hamburg, Germany

${ }^{28}$ University of Victoria, Department of Physics, P O Box 3055, Victoria BC V8W 3P6, Canada

${ }^{29}$ University of British Columbia, Department of Physics, Vancouver BC V6T 1Z1, Canada

${ }^{30}$ University of Alberta, Department of Physics, Edmonton AB T6G 2J1, Canada

${ }^{31}$ Duke University, Dept of Physics, Durham, NC 27708-0305, USA

${ }^{32}$ Research Institute for Particle and Nuclear Physics, H-1525 Budapest, P O Box 49, Hungary

${ }^{33}$ Institute of Nuclear Research, H-4001 Debrecen, P O Box 51, Hungary 
${ }^{34}$ Ludwigs-Maximilians-Universität München, Sektion Physik, Am Coulombwall 1, D-85748 Garching, Germany

${ }^{a}$ and at TRIUMF, Vancouver, Canada V6T 2A3

${ }^{b}$ and Royal Society University Research Fellow

${ }^{c}$ and Institute of Nuclear Research, Debrecen, Hungary

${ }^{d}$ and Department of Experimental Physics, Lajos Kossuth University, Debrecen, Hungary

${ }^{e}$ on leave of absence from the University of Freiburg 


\section{Introduction}

A direct search for charginos and neutralinos predicted in SUSY theories [1] is performed using the data collected with the OPAL detector at the centre-of-mass energies $(\sqrt{s})$ of $181-184 \mathrm{GeV}$ at the LEP $\mathrm{e}^{+} \mathrm{e}^{-}$collider at CERN. At these energies, chargino production cross-sections as large as $3.6 \mathrm{pb}$ for a mass of $85 \mathrm{GeV}$, together with the collected integrated luminosity of $57 \mathrm{pb}^{-1}$, lead to excellent discovery potential. This paper describes a chargino and neutralino search using the above data sample and an analysis which is improved relative to the one presented in a previous publication [2].

Previous searches for charginos and neutralinos have been performed by OPAL using data collected near the $\mathrm{Z}$ peak (LEP1), at $\sqrt{s}=130-136 \mathrm{GeV}$ [3], at $161 \mathrm{GeV}$ [4] and at 170 $172 \mathrm{GeV}$ [2], and by the other LEP collaborations [5] [6].

Charginos, $\tilde{\chi}_{j}^{ \pm}$, are the mass eigenstates formed by the mixing of the fields of the fermionic partners of the $\mathrm{W}$ boson (winos) and those of the charged Higgs bosons (charged higgsinos). Fermionic partners of the $\gamma, \mathrm{Z}$, and of the neutral Higgs bosons mix to form mass eigenstates called neutralinos, $\tilde{\chi}_{i}^{0}$. In each case, the index $j$ or $i$ is ordered by increasing mass. R-parity [7] conservation is assumed; therefore, the lightest supersymmetric particle (LSP) is stable. The LSP is usually considered to be the lightest neutralino, $\tilde{\chi}_{1}^{0}$, although it could be the scalar neutrino, $\tilde{\nu}$, if it is sufficiently light. The LSP is undetected due to its weakly interacting nature. The present analysis is valid for either choice of the LSP. In the Minimal Supersymmetric Standard Model (MSSM) there are two chargino mass eigenstates $\left(\tilde{\chi}_{1}^{ \pm}\right.$and $\left.\tilde{\chi}_{2}^{ \pm}\right)$and four neutralino mass eigenstates $\left(\tilde{\chi}_{1}^{0}, \tilde{\chi}_{2}^{0}, \tilde{\chi}_{3}^{0}\right.$ and $\left.\tilde{\chi}_{4}^{0}\right)$.

If charginos exist and are sufficiently light, they are pair-produced through a $\gamma$ or $\mathrm{Z}$ in the $s$-channel. For the wino component there is an additional production process through scalar electron-neutrino $\left(\tilde{\nu}_{\mathrm{e}}\right)$ exchange in the $t$-channel. The production cross-section is large unless the scalar neutrino (sneutrino) is light, in which case the cross-section is reduced by destructive interference between the $s$-channel $\mathrm{e}^{+} \mathrm{e}^{-}$annihilation to $\mathrm{Z}$ or $\gamma$ and $t$-channel $\tilde{\nu}_{\mathrm{e}}$ exchange diagrams [8, 9]. The details of chargino decay depend on the parameters of the mixing and the masses of the scalar partners of the ordinary fermions. The lightest chargino $\tilde{\chi}_{1}^{+}$can decay into $\tilde{\chi}_{1}^{0} \ell^{+} \nu$, or $\tilde{\chi}_{1}^{0} q \bar{q}^{\prime}$, via a $\mathrm{W}$ boson, scalar lepton $(\tilde{\ell}, \tilde{\nu})$ or scalar quark (squark, $\tilde{\mathrm{q}}$ ). In much of the MSSM parameter space, $\tilde{\chi}_{1}^{+}$decays via a $\mathrm{W}$ boson are dominant. Due to the energy and momentum carried away by the LSP (and possibly by neutrinos), the experimental signature for $\tilde{\chi}_{1}^{+} \tilde{\chi}_{1}^{-}$events is large missing energy and large missing momentum transverse to the beam axis. If the sneutrino is lighter than the chargino, the two-body decay $\tilde{\chi}_{1}^{+} \rightarrow \tilde{\nu} \ell^{+}$dominates. Special attention is paid to the case $m_{\tilde{\nu}} \approx m_{\tilde{\chi}_{1}^{ \pm}}$that would result in two low-momentum charged leptons.

Neutralino pairs $\left(\tilde{\chi}_{1}^{0} \tilde{\chi}_{2}^{0}\right)$ can be produced through an $s$-channel virtual Z, or by $t$-channel scalar electron (selectron, ê) exchange [10]. The MSSM prediction for the $\tilde{\chi}_{1}^{0} \tilde{\chi}_{2}^{0}$ production crosssection can vary significantly depending on the choice of MSSM parameters. It is typically a fraction of a picobarn and generally much lower than the cross-section for $\tilde{\chi}_{1}^{-} \tilde{\chi}_{1}^{+}$production. The $\tilde{\chi}_{2}^{0}$ will decay into $\tilde{\chi}_{1}^{0} \nu \bar{\nu}, \tilde{\chi}_{1}^{0} \ell^{+} \ell^{-}$or $\tilde{\chi}_{1}^{0} \mathrm{q} \overline{\mathrm{q}}$, through a $\mathrm{Z}^{(*)}$ boson, sneutrino, slepton, squark or a neutral SUSY Higgs boson $\left(\mathrm{h}^{0}\right.$ or $\left.\mathrm{A}^{0}\right)$. The decay via $\mathrm{Z}^{(*)}$ is the dominant mode in most of the parameter space. For the cases of $\tilde{\chi}_{2}^{0} \rightarrow \tilde{\chi}_{1}^{0} \ell^{+} \ell^{-}$or $\tilde{\chi}_{1}^{0} \mathrm{q} \overline{\mathrm{q}}$, this leads to an experimental signature consisting either of an acoplanar pair of particles or jets, or a monojet if the two jets in the final state have merged. The radiative decay process $\tilde{\chi}_{2}^{0} \rightarrow \tilde{\chi}_{1}^{0} \gamma$ is also possible [11] and can dominate for some regions of the parameter space. 
Motivated by Grand Unification and to simplify the physics interpretation, the Constrained Minimal Supersymmetric Standard Model (CMSSM) [8, 9, 10, 12] is used to guide the analysis but more general cases are also studied. In the CMSSM all the gauginos (SUSY partners of $U(1)_{Y}, S U(2)_{L}$ and $S U(3)_{c}$ gauge bosons) are assumed to have a common mass, $m_{1 / 2}$, at the grand unified (GUT) mass scale, and all the sfermions (SUSY partners of quarks and leptons) have a common mass, $m_{0}$, at the GUT mass scale.

In the CMSSM analyses reported here all possible cascade decay processes [10, 12] are taken into account. For example, if $\tilde{\chi}_{2}^{0}$ is lighter than $\tilde{\chi}_{1}^{+}$, the cascade decay of the chargino, $\tilde{\chi}_{1}^{+} \rightarrow \mathrm{ff} \tilde{\chi}_{2}^{0},\left(\tilde{\chi}_{2}^{0} \rightarrow \mathrm{ff} \tilde{\chi}_{1}^{0}\right.$, or $\left.\tilde{\chi}_{2}^{0} \rightarrow \gamma \tilde{\chi}_{1}^{0}\right)$, is possible円. The production of $\tilde{\chi}_{3}^{0}$ is also taken into account in the analysis. The experimental signatures for the $\tilde{\chi}_{1}^{0} \tilde{\chi}_{3}^{0}$ production are similar to those for $\tilde{\chi}_{1}^{0} \tilde{\chi}_{2}^{0}$, if $\tilde{\chi}_{3}^{0}$ decays into $\tilde{\chi}_{1}^{0} \mathrm{Z}^{(*)}$, or into $\tilde{\chi}_{1}^{0} \mathrm{~h}^{0}, \tilde{\chi}_{1}^{0} \mathrm{~A}^{0}$ or $\tilde{\chi}_{1}^{0} \gamma$.

This paper is organised as follows. The various event simulations which have been used are described in Section 2. Analyses of the possible signal topologies are discussed in Section 3 and results and physics interpretations, both model independent and based on the CMSSM, are given in Section 4.

\section{The OPAL Detector and Event Simulation}

\subsection{The OPAL Detector}

The OPAL detector is described in detail in [14. It is a multipurpose apparatus having nearly complete solid angle coverage ${ }^{2}$. The central tracking system consists of a silicon microvertex detector, a vertex drift chamber, a jet chamber and $z$-chambers. In the range $|\cos \theta|<0.73$, 159 points can be measured in the jet chamber along each track. At least 20 points on a track can be obtained over $96 \%$ of the full solid angle. The whole tracking system is located inside a $0.435 \mathrm{~T}$ axial magnetic field. A lead-glass electromagnetic (EM) calorimeter providing acceptance within $|\cos \theta|<0.984$ together with presamplers and time-of-flight scintillators is located both outside the magnet coil and at the front of each endcap. The magnet return yoke is instrumented for hadron calorimetry (HCAL), giving a polar angle coverage of $|\cos \theta|<0.99$, and is surrounded by external muon chambers. The forward detectors (FD) and silicon-tungsten calorimeters (SW) located on both sides of the interaction point measure the luminosity and complete the geometrical acceptance down to $24 \mathrm{mrad}$ in polar angle. The small gap between the endcap EM calorimeter and FD is filled by an additional electromagnetic calorimeter, called the gamma-catcher (GC).

\subsection{Event Simulation}

The DFGT generator [15] is used to simulate signal events. It includes spin correlations and allows for a proper treatment of both the $\mathrm{W}$ boson and the $\mathrm{Z}$ boson width effects in the chargino

\footnotetext{
${ }^{1}$ Pair production of $\tilde{\chi}_{2}^{0} \tilde{\chi}_{2}^{0}$ is also possible, but direct searches for this channel with the decays $\tilde{\chi}_{2}^{0} \rightarrow \tilde{\chi}_{1}^{0} \mathrm{Z}^{(*)}$ would contribute negligibly to the overall limits placed on the CMSSM parameter space, and direct searches for this mode are not made in this analysis. The process $\tilde{\chi}_{2}^{0} \tilde{\chi}_{2}^{0} \rightarrow \gamma \tilde{\chi}_{1}^{0} \gamma \tilde{\chi}_{1}^{0}$ is taken into account in the CMSSM limits calculations using the experimental results of another OPAL analysis 13 .

2 A right-handed coordinate system is adopted, where the $x$-axis points to the centre of the LEP ring, and positive $z$ is along the electron beam direction. The angles $\theta$ and $\phi$ are the polar and azimuthal angles, respectively.
} 
and heavy neutralino decays. The generator includes initial-state radiation and uses the JETSET 7.4 package [16] for the hadronisation of the quark-antiquark system in the hadronic decays of charginos and neutralinos. SUSYGEN [17] is used to calculate the branching fractions for the CMSSM interpretation of the analysis. The most important parameters influencing the chargino detection efficiency are the mass of the lightest chargino, $m_{\tilde{\chi}_{1}^{+}}$, and the mass difference between the lightest chargino and the lightest neutralino, $\Delta M_{+} \equiv m_{\tilde{\chi}_{1}^{+}}-m_{\tilde{\chi}_{1}^{0}} \cdot \tilde{\chi}_{1}^{+} \tilde{\chi}_{1}^{-}$events are generated for 78 points in the $\left(m_{\tilde{\chi}_{1}^{+}}, \Delta M_{+}\right)$plane, for $m_{\tilde{\chi}_{1}^{+}}$between $50 \mathrm{GeV}$ and $90 \mathrm{GeV}$ and $\Delta M_{+}$between $3 \mathrm{GeV}$ and $m_{\tilde{\chi}_{1}^{+}}$. At each point 1000 events for the decay $\tilde{\chi}_{1}^{+} \rightarrow \tilde{\chi}_{1}^{0} \mathrm{~W}^{*+}$ are generated. For the two-body decays of the chargino $\left(\tilde{\chi}_{1}^{+} \rightarrow \tilde{\nu} \ell^{+}\right), 43$ points of 1000 events are generated in the $\left(m_{\tilde{\chi}_{1}^{+}}, m_{\tilde{\nu}}\right)$ plane, for $m_{\tilde{\chi}_{1}^{+}}$between $45 \mathrm{GeV}$ and $90 \mathrm{GeV}$ and $m_{\tilde{\nu}}$ between $1.5 \mathrm{GeV}$ and $m_{\tilde{\chi}_{1}^{+}}-35 \mathrm{GeV}$. For the $\tilde{\chi}_{1}^{0} \tilde{\chi}_{2}^{0}$ production, $m_{\tilde{\chi}_{2}^{0}}$ and $\Delta M_{0} \equiv m_{\tilde{\chi}_{2}^{0}}-m_{\tilde{\chi}_{1}^{0}}$ are the main parameters affecting the efficiency. The $\tilde{\chi}_{1}^{0} \tilde{\chi}_{2}^{0}$ events are generated in 75 points of the $\left(m_{\tilde{\chi}_{2}^{0}}, \Delta M_{0}\right)$ plane, for $m_{\tilde{\chi}_{1}^{0}}+m_{\tilde{\chi}_{2}^{0}}$ between $100 \mathrm{GeV}$ and $180 \mathrm{GeV}$ and $\Delta M_{0}$ between $3 \mathrm{GeV}$ and $m_{\tilde{\chi}_{2}^{0}}$. For the cascade decay of $\tilde{\chi}_{1}^{ \pm}, 55$ points are generated varying $m_{\tilde{\chi}_{2}^{0}}, m_{\tilde{\chi}_{1}^{0}}$, and the branching fractions for $\tilde{\chi}_{1}^{+} \rightarrow \mathrm{f} \overline{\mathrm{f}} \tilde{\chi}_{2}^{0}$ and $\tilde{\chi}_{2}^{0} \rightarrow \tilde{\chi}_{1}^{0} \gamma$.

The sources of background to the chargino and neutralino signals are two-photon, lepton pairs, multihadronic and four-fermion processes. Two-photon processes are the most important background for the case of small $\Delta M_{+}$and small $\Delta M_{0}$ where the visible energy and momentum transverse to the beam direction for signal and two-photon events are comparatively small. The Monte Carlo generators PHOJET [18] (for $Q^{2}<4.5 \mathrm{GeV}^{2}$ ) and HERWIG [19] (for $Q^{2} \geq 4.5 \mathrm{GeV}^{2}$ ) are used to simulate hadronic events from two-photon processes. The Vermaseren [20] program is used to simulate leptonic two-photon processes $\left(\mathrm{e}^{+} \mathrm{e}^{-} \mathrm{e}^{+} \mathrm{e}^{-}, \mathrm{e}^{+} \mathrm{e}^{-} \mu^{+} \mu^{-}\right.$ and $\mathrm{e}^{+} \mathrm{e}^{-} \tau^{+} \tau^{-}$). Four-fermion processes are simulated using the grc4f [21] generator, which takes into account all interferences. The dominant contributions are $\mathrm{W}^{+} \mathrm{W}^{-}, \mathrm{We} \nu, \gamma^{*} \mathrm{Z}$ and $\mathrm{ZZ}^{(*)}$ events, which have topologies very similar to that of the signal. Additional samples of $\mathrm{e}^{+} \mathrm{e}^{-} \mathrm{e}^{+} \mathrm{e}^{-}, \mathrm{e}^{+} \mathrm{e}^{-} \mu^{+} \mu^{-}$and $\mathrm{e}^{+} \mathrm{e}^{-} \tau^{+} \tau^{-}$processes which are not covered by the Vermaseren program are generated using grc4f. Lepton pairs are generated using the KORALZ [22] generator for $\tau^{+} \tau^{-}(\gamma)$ and $\mu^{+} \mu^{-}(\gamma)$ events, and the BHWIDE [23] program for $\mathrm{e}^{+} \mathrm{e}^{-} \rightarrow \mathrm{e}^{+} \mathrm{e}^{-}(\gamma)$ events. Multihadronic $(\mathrm{q} \overline{\mathrm{q}}(\gamma))$ events are simulated using PYTHIA [16].

Generated signal and background events are processed through the full simulation of the OPAL detector [24] and the same event analysis chain was applied to the simulated events as to the data.

\section{Data Analysis}

The analysis is performed on data collected during the 1997 run of LEP at $\sqrt{s}=181-184 \mathrm{GeV}$. The luminosity weighted average of $\sqrt{s}$ is $182.7 \mathrm{GeV}$.

Data are used from runs in which all the subdetectors relevant to this analysis were fully operational, corresponding to an integrated luminosity of $56.75 \pm 0.27 \mathrm{pb}^{-1}$. The luminosity is measured using small-angle Bhabha scattering events detected in the silicon-tungsten calorimeter.

\footnotetext{
${ }^{3}$ Most of the data was taken at $182.7 \mathrm{GeV}, 3.3 \%$ of the data was taken at $183.8 \mathrm{GeV}, 6.1 \%$ at $181.8 \mathrm{GeV}$ and $0.6 \%$ at less than $181.0 \mathrm{GeV}$. The error of $\sqrt{s}$ from the beam energy uncertainty is $0.03 \mathrm{GeV}$.
} 
The following preselection cuts are applied to all data to select well measured events: (1) the number of charged tracks is required to be at least two; (2) the event transverse momentum relative to the beam direction is required to be larger than $1.8 \mathrm{GeV}$; (3) the total energy deposit in each side of the SW, FD and GC detectors has to be smaller than 2, 2 and $5 \mathrm{GeV}$, respectively; (4) the visible invariant mass of the event has to exceed $2 \mathrm{GeV}$; (5) the maximum EM cluster energy and the maximum charged track momentum has to be smaller than $130 \%$ of the beam energy to reject badly reconstructed events; and (6) the absolute mean of the impact parameters (with respect to the beam spot position) of the reconstructed charged tracks are required to be smaller than $1 \mathrm{~cm}$ for the high multiplicity analyses to reject background from beam-gas and beam-wall interactions.

\subsection{Detection of Charginos}

To obtain optimal performance the event sample is divided exclusively into three categories, motivated by the topologies expected from chargino events. Separate analyses are applied to the preselected events in each category:

(A) $N_{\text {ch }}>4$ and no isolated leptons, where $N_{\text {ch }}$ is the number of charged tracks: when both $\tilde{\chi}_{1}^{+}$and $\tilde{\chi}_{1}^{-}$decay hadronically, signal events tend to fall into this category for modest and large values of $\Delta M_{+}\left(\equiv m_{\tilde{\chi}_{1}^{+}}-m_{\tilde{\chi}_{1}^{0}}\right)$.

(B) $N_{\text {ch }}>4$ and at least one isolated lepton: if only one of the $\tilde{\chi}_{1}^{ \pm}$decays leptonically, signal events tend to fall into this category.

(C) $N_{\mathrm{ch}} \leq 4$ : events tend to fall into this category if $\Delta M_{+}$is small or if both charginos decay leptonically.

The isolated lepton selection criteria are similar to those described in Ref. [2]. Electrons are selected if they satisfy either of the two identification methods described in [25, 26] and muons are identified using three methods [27, 28, 29]. The momentum of the electron or muon candidate is required to be larger than $2 \mathrm{GeV}$. In order to identify a tau lepton, events are reconstructed using the Durham jet algorithm with a jet resolution parameter of $3 \mathrm{GeV}^{2}$. A reconstructed jet is identified as a tau decay if there are only one or three charged tracks in the jet, if the momentum sum of the charged tracks is larger than $2.0 \mathrm{GeV}$, if the invariant mass of the charged particles in the jet is smaller than $1.5 \mathrm{GeV}$ and if the invariant mass of the jet is smaller than $2.0 \mathrm{GeV}$. The identified lepton is defined to be isolated if the energy within a cone of half-angle $20^{\circ}$ around the electron, muon or tau candidate is less than $2 \mathrm{GeV}$.

The fraction of $\tilde{\chi}_{1}^{+} \tilde{\chi}_{1}^{-}$events falling into category $(\mathrm{A})$ is about $35-50 \%$ for most $\Delta M_{+}$values. This fraction drops to less than $15 \%$ if $\Delta M_{+}$is smaller than $5 \mathrm{GeV}$, since the average charged track multiplicity of the events is smaller. Similarly, the fraction of events falling into category (B) is also about $35-50 \%$ for most $\Delta M_{+}$values and is less than $10 \%$ if $\Delta M_{+}$is smaller than $5 \mathrm{GeV}$. When $\Delta M_{+}$is smaller than $10 \mathrm{GeV}$, the fraction of events falling into category $(\mathrm{C})$ is greater than about $50 \%$. If $\Delta M_{+}$is larger than $20 \mathrm{GeV}$, this fraction is about $10 \%$.

Since the chargino event topology mainly depends on the difference between the chargino mass and the lightest neutralino mass, different selection criteria are applied to four $\Delta M_{+}$ regions: 
(I) $\Delta M_{+} \leq 10 \mathrm{GeV}$,

(II) $10 \mathrm{GeV}<\Delta M_{+} \leq m_{\tilde{\chi}_{1}^{+}} / 2$,

(III) $m_{\tilde{\chi}_{1}^{+}} / 2<\Delta M_{+} \leq m_{\tilde{\chi}_{1}^{+}}-20 \mathrm{GeV}$,

(IV) $m_{\tilde{\chi}_{1}^{+}}-20 \mathrm{GeV}<\Delta M_{+} \leq m_{\tilde{\chi}_{1}^{+}}$.

In region I, the main background comes from two-photon processes. Background from fourfermion processes (mainly $\mathrm{W}^{+} \mathrm{W}^{-}$) can be safely rejected without sacrificing signal detection efficiency. In regions II and III, the main background comes from four-fermion processes $\left(\mathrm{W}^{+} \mathrm{W}^{-}\right.$, single $\mathrm{W}$ and $\left.\gamma^{*} Z^{(*)}\right)$. In these regions the background level is modest. In region IV the $\mathrm{W}^{+} \mathrm{W}^{-}$ background is large and dominant. Since the $\mathrm{W}^{+} \mathrm{W}^{-}$background is very severe in the region of $\Delta M_{+}>85 \mathrm{GeV}$ where the chargino decays via an on-mass-shell $\mathrm{W}$-boson, a special analysis (allowing relatively high background and demanding higher signal efficiency) is applied to improve the sensitivity to the chargino signal. Overlap between this analysis and the region IV standard analysis is avoided by selecting the analysis which minimises the expected crosssection limit calculated with only the expected background number. This generally results in the special analysis being applied for $m_{\tilde{\chi}_{1}^{+}}>85 \mathrm{GeV}$ and $\Delta M_{+} \gtrsim 85 \mathrm{GeV}$. If $m_{\tilde{\nu}}$ is lighter than $\tilde{\chi}_{1}^{+}$, the two-body decays of the chargino $\left(\tilde{\chi}_{1}^{+} \rightarrow \tilde{\nu} \ell^{+}\right)$may dominate over the three-body decays via a virtual W. A special analysis, optimised for this case, is also performed in category (C).

For each region a single set of cut values is determined which minimises the expected limit on the signal cross-section at 95\% C.L. using Ref. [30]. For this procedure, it is assumed that the distribution of observed candidates arises from the expected number of background events and therefore the choice of cuts is independent of the number of candidates actually observed.

The efficiency for an arbitrary choice of $m_{\tilde{\chi}_{1}^{+}}$and $m_{\tilde{\chi}_{1}^{0}}$ is obtained by interpolation using a polynomial fit to the efficiencies determined from the Monte Carlo for each category in each $\Delta M_{+}$region.

\subsubsection{Analysis (A) $\left(N_{\mathrm{ch}}>4\right.$ without isolated leptons)}

For modest and large values of $\Delta M_{+}$, if both $\tilde{\chi}_{1}^{+}$and $\tilde{\chi}_{1}^{-}$decay hadronically, signal events tend to fall into category (A). The variables used in the selection criteria and their cut values optimised in each $\Delta M_{+}$region are listed in Table 1 .

After the preselection, the cuts on $E_{\mathrm{fwd}} / E_{\mathrm{vis}},\left|\cos \theta_{\text {miss }}\right|,\left|P_{z}\right|$ and $\left|P_{z}\right| / E_{\mathrm{vis}}$, where $E_{\mathrm{vis}}$ is the total visible energy of the event, $E_{\text {fwd }}$ is the visible energy in the region of $|\cos \theta|>0.9, \theta_{\text {miss }}$ is the polar angle of the missing momentum and $P_{z}$ is the visible momentum along the beam axis, are applied to reduce background from two-photon and $Z^{0}$ radiative return processes. Most of the remaining background from two-photon processes is rejected by the cuts on $P_{\mathrm{t}}$ and $P_{\mathrm{t}}^{\mathrm{HCAL}}$, the transverse momentum of the event measured without using the hadron calorimeter and using the hadron calorimeter, respectively. In region $\mathrm{I}$, the upper cut on $P_{\mathrm{t}}^{\mathrm{HCAL}}$ is determined from the range of the signal $P_{\mathrm{t}}^{\mathrm{HCAL}}$ distribution; it reduces the background from $\mathrm{W}^{+} \mathrm{W}^{-}$and We $\nu$ events.

The number of jets $\left(N_{\text {jet }}\right)$ is reconstructed using the Durham jet algorithm with jet resolution parameter $y_{\text {cut }}=0.005$. With the $N_{\text {jet }}$ cut, small invariant mass monojet events from the process 


\begin{tabular}{|c|c|c|c|c|}
\hline Region & I & II & III & IV \\
\hline$\overline{\overline{E_{\text {fwd }}} / E_{\mathrm{vis}}}$ & \multicolumn{4}{|c|}{$<0.2$} \\
\hline$\left|\cos \theta_{\text {miss }}\right|$ & \multicolumn{4}{|c|}{$<0.85$} \\
\hline$\left|P_{z}\right| \mathrm{GeV}$ & \multicolumn{4}{|c|}{$<30$} \\
\hline$\left|P_{z}\right| / E_{\mathrm{vis}}$ & \multicolumn{4}{|c|}{$<0.7$} \\
\hline$P_{\mathrm{t}} \mathrm{GeV}$ & \multicolumn{4}{|c|}{$>6$} \\
\hline$P_{\mathrm{t}}^{\mathrm{HCAL}} \mathrm{GeV}$ & {$[6,30]$} & & $>6$ & \\
\hline$N_{\text {jet }}$ & \multicolumn{2}{|c|}{$[2,5]$} & \multicolumn{2}{|c|}{$[3,5]$} \\
\hline$\left|\cos \theta_{\mathrm{j}}\right|$ & \multicolumn{4}{|c|}{$<0.90$} \\
\hline$\phi_{\text {acop }}^{\circ}$ & \multicolumn{2}{|c|}{$>15\left(10^{*}\right)$} & \multicolumn{2}{|c|}{$>15$} \\
\hline $\begin{array}{l}\text { if } M_{\text {vis }}>90, \\
p_{\max } \mathrm{GeV}\end{array}$ & \multicolumn{2}{|c|}{ 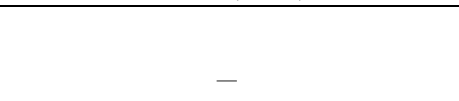 } & \multicolumn{2}{|c|}{$<20$} \\
\hline$M_{\mathrm{vis}} \mathrm{GeV}$ & $<60$ & {$[5,80]$} & {$[5,130]$} & {$[5,150]$} \\
\hline if $N_{\ell^{\prime}}=1$ & & & & \\
\hline$M_{\mathrm{had}^{\prime}} \mathrm{GeV}$ & $<30$ & $<50$ & $<60$ & $\{60,90\}$ \\
\hline$E_{\ell^{\prime}} \mathrm{GeV}$ & \multicolumn{3}{|c|}{$<30$} & $<35$ \\
\hline$E_{1} \mathrm{GeV}$ & - & {$[2,35]$} & {$[2,50]$} & {$[2,55]$} \\
\hline$E_{2} \mathrm{GeV}$ & - & {$[2,25]$} & {$[2,50]$} & {$[2,55]$} \\
\hline $\begin{array}{l}\text { if } N_{\text {jet }}=3 \\
\left|P_{z}\right| \mathrm{GeV}\end{array}$ & \multicolumn{2}{|c|}{-} & \multicolumn{2}{|c|}{$<10^{* *}$} \\
\hline \multicolumn{5}{|l|}{ background } \\
\hline$\gamma \gamma$ & 0.23 & 0.11 & 0.11 & 0.11 \\
\hline$\ell^{+} \ell^{-}(\gamma)$ & 0.02 & 0.02 & 0.01 & 0.01 \\
\hline $\mathrm{q} \overline{\mathrm{q}}(\gamma)$ & 0.10 & 0.11 & 0.40 & 0.82 \\
\hline $4 \mathrm{f}$ & 0.67 & 1.09 & 5.89 & 8.43 \\
\hline$\overline{\text { total bkg. }}$ & $\bar{~} 1.01 \pm 0.17$ & 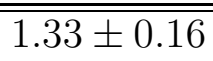 & $6.42 \pm 0.29$ & $\overline{\overline{9} .38 \pm 0.34}$ \\
\hline observed & $\begin{array}{ll} \\
1\end{array}$ & $\begin{array}{ll} \\
1\end{array}$ & $\begin{array}{ll} \\
4\end{array}$ & $\begin{array}{ll}7\end{array}$ \\
\hline
\end{tabular}

Table 1: The list of selection criteria for category (A). The numbers of background events expected for the integrated luminosity of $57 \mathrm{pb}^{-1}$, for various Standard Model processes, and the total number of background events expected as well as the observed number of events in each $\Delta M_{+}$region are also listed. If $N_{\text {jet }}=2, \phi_{\text {acop }}$ is required to be larger than $10^{\circ}$ (as indicated by ${ }^{*}$ ). $N_{\text {jet }}=[2,5]$ means that $2 \leq N_{\text {jet }} \leq 5 . \quad M_{v i s}=\{60,90\}$ means that $M_{\text {vis }}<60$ or $M_{\text {vis }}>90 \mathrm{GeV}$. If $N_{\text {jet }}=3$ and $70<M_{\text {vis }}<95 \mathrm{GeV}$ in region III and IV, $\left|P_{z}\right|$ is required to be smaller than $10 \mathrm{GeV}$ (as indicated by $\left.{ }^{* *}\right)$. The errors in the total background include only the statistical errors of simulated background events.

$\gamma^{*} Z^{(*)} \rightarrow \mathrm{q} \overline{\mathrm{q}} \nu \bar{\nu}$ are rejected for regions I and II, and background from $\mathrm{q} \overline{\mathrm{q}}(\gamma)$ and single W events is reduced in regions III and IV.

After the above cuts, to calculate the acoplanarity angle $\left(\phi_{\text {acop }}\right)$, the events are forced into two jets, again using the Durham jet algorithm. The polar angle of each jet, $\theta_{\mathbf{j}}(\mathrm{j}=1,2)$, is required to be far from the beam axis. This cut ensures a good measurement of $\phi_{\text {acop }}$ and further reduces the background from the $\mathrm{q} \overline{\mathrm{q}}(\gamma)$ and two-photon processes. To select signals with 
a large amount of missing momentum due to the two invisible neutralinos and to reduce $q \bar{q}(\gamma)$ background, the acoplanarity angle is required to be large. The acoplanarity angle distributions for region II are shown in Fig. 1 before application of this cut.

For events with an observed invariant mass $\left(M_{\text {vis }}\right)$ greater than $90 \mathrm{GeV}$, a cut is applied on the maximum track momentum $\left(p_{\max }\right)$ in regions III and IV. This cut reduces $\mathrm{W}^{+} \mathrm{W}^{-} \rightarrow \ell \nu \mathrm{q} \overline{\mathrm{q}}^{\prime}$ events where the lepton $\ell$ overlaps with a hadronic jet. The $M_{\text {vis }}$ cut is optimised for each $\Delta M_{+}$region as shown in Table 1. The distributions of the observed invariant mass are shown in Fig. 2 for region II, after the $\phi_{\text {acop }}$ cut.

If a lepton $\left(\ell^{\prime}\right)$ is found with an algorithm based on the looser isolation condition described in Ref. [2], the lepton energy $E_{\ell^{\prime}}$ and the invariant mass calculated without the lepton, $M_{\text {had }^{\prime}}$, must be smaller than the values expected for $\mathrm{W}^{+} \mathrm{W}^{-} \rightarrow \ell \nu \mathrm{q} \overline{\mathrm{q}}^{\prime}$ events.

The backgrounds from $\mathrm{W}^{+} \mathrm{W}^{-}$events and single $\mathrm{W}$ events are efficiently suppressed by requiring that the highest energy $\left(E_{1}\right)$ and the second highest energy $\left(E_{2}\right)$ of the jets (reconstructed with $\left.y_{\text {cut }}=0.005\right)$ are smaller than the typical jet energy expected for the $\mathrm{W}^{+} \mathrm{W}^{-}$ background. In region III and IV three-jet events with $\left|P_{Z}\right|<10 \mathrm{GeV}$ are rejected if $M_{\text {vis }}$ is close to the $\mathrm{W}$ mass. This cut reduces the $\mathrm{W}^{+} \mathrm{W}^{-} \rightarrow \tau \nu \mathrm{q} \overline{\mathrm{q}}^{\prime}$ background with small $\tau$ decay product energy.

The numbers of background events expected from the four different sources, for each $\Delta M_{+}$ region, are given in Table 1. Typical detection efficiencies for $\tilde{\chi}_{1}^{+} \tilde{\chi}_{1}^{-}$events are shown in Fig. 9a.

A special analysis is applied in the region of $\Delta M_{+} \gtrsim 85 \mathrm{GeV}$, since the event topology of the signal is very similar to that of $\mathrm{W}^{+} \mathrm{W}^{-} \rightarrow 4$ jets. This similarity is due to the small missing momentum taken by the low mass neutralinos. After selecting well contained events with the cuts $\left|\cos \theta_{\text {miss }}\right|<0.95, E_{\mathrm{fwd}} / E_{\mathrm{vis}}<0.25$ and $\left|P_{z}\right|<30 \mathrm{GeV}$, multijet events with large visible energy are selected $\left(N_{\text {jet }} \geq 4\right.$ and $\left.100<E_{\text {vis }}<170 \mathrm{GeV}\right)$. To select a clear 4-jet topology, $y_{34} \geq 0.0075, y_{23} \geq 0.04$ and $y_{45} \leq 0.0015$ are required, where $y_{\{n\}\{n+1\}}$ is defined as the minimum $y_{\text {cut }}$ value at which the event is reconstructed as having $n$-jets. If there is a jet which consists of a single $\gamma$ with energy greater than $50 \mathrm{GeV}$, events are considered to be $\gamma q \bar{q} g$ and are rejected. The maximum track momentum is required to be smaller than $40 \mathrm{GeV}$ to reduce background from $\mathrm{W}^{+} \mathrm{W}^{-} \rightarrow \ell \nu \mathrm{q} \overline{\mathrm{q}}^{\prime}$ events where $\ell$ overlaps with a hadronic jet. The number of selected events is 29 , while the number expected from background processes is $24.7 \pm 0.5(0.0$ from $\gamma \gamma, 0.0$ from $\ell^{+} \ell^{-}(\gamma), 6.4$ from $\mathrm{q} \overline{\mathrm{q}}(\gamma)$ and 18.3 from four fermion final states). The signal efficiency is $26-33 \%$ for $m_{\tilde{\chi}_{1}^{+}} \geq 85 \mathrm{GeV}$ and $\Delta M_{+} \geq 85 \mathrm{GeV}$.

\subsubsection{Analysis (B) $\left(N_{\mathrm{ch}}>4\right.$ with isolated leptons $)$}

$\tilde{\chi}_{1}^{+} \tilde{\chi}_{1}^{-}$events in which one of the $\tilde{\chi}_{1}^{ \pm}$decays leptonically tend to fall into category (B). The variables used in the selection and their cut values in each $\Delta M_{+}$region are listed in Table 2. The signal events are selected with the following criteria:

Cuts on $\left|\cos \theta_{\text {miss }}\right|$ and $E_{\text {fwd }} / E_{\text {vis }}$ are made to reject background from two-photon processes and $q \bar{q}(\gamma)$ events. Large transverse momenta are then required to further reduce the contribution from two-photon events. The distributions of $P_{\mathrm{t}}$ are shown in Fig. 3 after the $\left|\cos \theta_{\text {miss }}\right|$ cut. To calculate the acoplanarity angle, events are forced into two jets. A large acoplanarity angle of the two jets is then required to further suppress the two-photon background.

In order to reject $\mathrm{W}^{+} \mathrm{W}^{-} \rightarrow \ell \nu \mathrm{q} \overline{\mathrm{q}}^{\prime}$ background, various cuts are applied: the momentum of isolated leptons should be small enough; the invariant mass of the event calculated excluding 


\begin{tabular}{|c|c|c|c|c|}
\hline Region & $\mathrm{I}$ & II & III & IV \\
\hline$\overline{\overline{E_{\text {fwd }}} / E_{\mathrm{vis}}}$ & $<0.15$ & $\overline{<<0.2}$ & \multicolumn{2}{|c|}{$<<0.3$} \\
\hline$\left|\cos \theta_{\text {miss }}\right|$ & \multicolumn{4}{|c|}{$<0.9$} \\
\hline$P_{\mathrm{t}} \mathrm{GeV}$ & $>4$ & $>5$ & $>7$ & $>8$ \\
\hline$P_{\mathrm{t}}^{\mathrm{HCAL}} \mathrm{GeV}$ & $>4$ & $>5$ & $>7$ & $>8$ \\
\hline$\left|\cos \theta_{\mathrm{j}}\right|$ & \multicolumn{4}{|c|}{$<0.95$} \\
\hline$\phi_{\text {acop }}^{\circ}$ & \multicolumn{2}{|c|}{$>20$} & \multicolumn{2}{|c|}{$>15$} \\
\hline$M_{\text {had }} \mathrm{GeV}$ & $<30$ & $<60$ & {$[5,60]$} & {$[5,65]$} \\
\hline$p_{\ell} \mathrm{GeV}$ & $<20$ & \multicolumn{2}{|c|}{$<45^{*}$} & {$[4,50]$} \\
\hline$M_{\text {vis }} \mathrm{GeV}$ & $<50$ & \multicolumn{2}{|c|}{$<80$} & $<80^{* *}$ \\
\hline$\overline{\text { background }}$ & & & & \\
\hline$\gamma \gamma$ & 0.84 & 0.06 & 0.06 & 0.06 \\
\hline$\ell^{+} \ell^{-}(\gamma)$ & 0.02 & 0.10 & 0.13 & 0.18 \\
\hline $\bar{q} \overline{\mathrm{q}}(\gamma)$ & 0.00 & 0.02 & 0.11 & 0.11 \\
\hline $4 \mathrm{f}$ & 0.33 & 1.18 & 1.27 & 3.33 \\
\hline total bkg & $1.20 \pm 0.49$ & $1.36 \pm 0.13$ & $1.56 \pm 0.14$ & $3.68 \pm 0.21$ \\
\hline observed & $\overline{0}$ & 1 & $\overline{1}$ & $\overline{3}$ \\
\hline
\end{tabular}

Table 2: The list of selection criteria for category $(\mathrm{B})$. The $p_{\ell}$ cut for regions II and III (as indicated by $*$ ) are a function of $M_{\text {had }}\left(p_{\ell}<4.5 M_{\text {had }}\right.$ if $M_{\text {had }}<10 \mathrm{GeV}, p_{\ell} \leq 45 \mathrm{GeV}$ if $10 \leq M_{\text {had }} \leq 50 \mathrm{GeV}$, $p_{\ell} \leq-4.5\left(M_{\text {had }}-60 \mathrm{GeV}\right)$ if $\left.M_{\text {had }}>50 \mathrm{GeV}\right)$. The $M_{\text {vis }}$ cut in region IV (as indicated by $* *$ ) is only applied if $M_{\text {had }}>55 \mathrm{GeV}$. The numbers of background events expected for the integrated luminosity of $57 \mathrm{pb}^{-1}$ for various Standard Model processes and the total number of background events expected as well as the observed number of events in each $\Delta M_{+}$region are also listed. The errors in the total background include only the statistical error of simulated background events.

the highest momentum isolated lepton $\left(M_{\text {had }}\right)$ is required to be smaller than the W mass; in the small $\Delta M_{+}$region the upper cut value of $M_{\text {had }}$ is reduced, since signal events are concentrated only in the small mass region. The distribution of $M_{\text {had }}$ after the $\phi_{\text {acop }}$ cut is shown in Fig. 目 for region III. As shown in this figure, most of the $\mathrm{W}^{+} \mathrm{W}^{-}$background events are rejected. Futhermore, $\mathrm{We} \nu$ candidate events in which a fake lepton is found in the $\mathrm{W} \rightarrow \mathrm{q} \overline{\mathrm{q}}^{\prime}$ decay are further reduced by the $M_{\text {vis }}$ cut. Typical detection efficiencies for $\tilde{\chi}_{1}^{+} \tilde{\chi}_{1}^{-}$events are shown in Fig. 9 a.

A special analysis is applied in the region of $\Delta M_{+} \gtrsim 85 \mathrm{GeV}$ due to the large $\mathrm{W}^{+} \mathrm{W}^{-}$ background. The selection criteria are identical to those in region IV up to the $\phi_{\text {acop }}$ cut as listed in the upper part of Table 2. To reject some $\mathrm{W}^{+} \mathrm{W}^{-} \rightarrow \ell \nu \mathrm{qq} \overline{\mathrm{q}}^{\prime}$ events, while keeping a good signal efficiency, $M_{\text {had }}$ is required to be between 50 and $90 \mathrm{GeV}$, and $M_{\text {vis }}$ to be between 80 and $130 \mathrm{GeV}$. The invariant mass of the missing four-momentum and the four-momentum of the highest energy isolated lepton must lie between 90 and $130 \mathrm{GeV}$. The number of observed events with these criteria is 28 , while the number of expected background is $31.8 \pm 0.6$, almost all from four-fermion processes. The signal efficiency is $30-41 \%$ for $m_{\tilde{\chi}_{1}^{+}} \geq 85 \mathrm{GeV}$ and $\Delta M_{+} \geq 85 \mathrm{GeV}$. 


\subsubsection{Analysis (C) $\left(N_{\mathrm{ch}} \leq 4\right)$}

Events in which both charginos decay leptonically as well as a large fraction of events for small $\Delta M_{+}$tend to fall into category (C). This analysis is especially important for the region of $\Delta M_{+} \leq 5 \mathrm{GeV}$. Because the background varies significantly with $\Delta M_{+}$in region $\mathrm{I}$, this region has been split into 2 sub-regions $(\mathrm{a}, \mathrm{b})$. The cut variables and the cut values for each region and sub-region are listed in Table 3 .

\begin{tabular}{|c|c|c|c|c|c|}
\hline Region & $\mathrm{I}$ & & II & III & IV \\
\hline Sub-region & $\mathrm{a}$ & $\mathrm{b}$ & & & \\
\hline $\begin{array}{l}N_{\mathrm{ch}} \\
\Sigma O_{i}\end{array}$ & \multicolumn{5}{|c|}{$\begin{array}{c}{[2,4]} \\
0\end{array}$} \\
\hline $\begin{array}{l}E_{\mathrm{j}} \mathrm{GeV} \\
N_{\mathrm{ch}, \mathrm{j}} \\
\left|Q_{\mathrm{j}}\right|\end{array}$ & \multicolumn{5}{|c|}{$\geq 1.5$} \\
\hline $\begin{array}{l}\text { for } \phi_{\text {acop }}^{\circ} \\
a_{\mathrm{t}} / E_{\text {beam }} \\
P_{\mathrm{t}} / E_{\text {beam }} \\
\left|\cos \theta_{a}\right|\end{array}$ & \multicolumn{2}{|c|}{$\begin{array}{c}\leq 70 \\
>0.030 \\
>0.030 \\
<0.975\end{array}$} & & $\begin{array}{l}- \\
- \\
- \\
-\end{array}$ & \\
\hline for $\phi_{\text {acop }}^{\circ}$ & \multicolumn{2}{|c|}{$>70$} & \multicolumn{3}{|c|}{-} \\
\hline$P_{\mathrm{t}} / E_{\text {beam }}$ & $>0.040$ & $>0.050$ & $>0.075$ & $>0.095$ & $>0.100$ \\
\hline$\left|\cos \theta_{\text {miss }}\right|$ & \multicolumn{3}{|c|}{$<0.90$} & \multicolumn{2}{|c|}{$<0.97$} \\
\hline$\Delta \phi_{\left(\vec{P}_{\text {miss }}, \mu\right)} \mathrm{rad}$ & \multicolumn{5}{|c|}{$>1.0$} \\
\hline$\left|\cos \theta_{\mathrm{j}}\right|$ & \multicolumn{2}{|c|}{$<0.85$} & $<0.95$ & \multicolumn{2}{|c|}{$<0.97$} \\
\hline$\phi_{\text {acop }}^{\circ}$ & \multicolumn{2}{|c|}{$[20,150]$} & \multicolumn{3}{|c|}{$>20$} \\
\hline$M_{\text {vis }} \mathrm{GeV}$ & $<10.0$ & $<15.0$ & $<30.0$ & $<40.0$ & $<60.0$ \\
\hline$\overline{E_{1} / E_{\text {beam }}}$ & $<0.15$ & $<0.22$ & $<0.30$ & \multicolumn{2}{|c|}{$<1.00$} \\
\hline background & & & & & \\
\hline$\gamma \gamma$ & 1.99 & 0.68 & 2.04 & 1.53 & 1.31 \\
\hline$\ell^{+} \ell^{-}(\gamma)$ & 0.02 & 0.03 & 0.24 & 0.83 & 1.50 \\
\hline $\mathrm{q} \overline{\mathrm{q}}(\gamma)$ & 0.00 & 0.00 & 0.01 & 0.01 & 0.01 \\
\hline 4f & 0.33 & 0.50 & 2.47 & 14.70 & 27.04 \\
\hline total bkg. & $2.33 \pm 0.36$ & $1.21 \pm 0.21$ & $4.77 \pm 0.38$ & $17.08 \pm 0.48$ & $29.86 \pm 0.55$ \\
\hline observed & 2 & 0 & 3 & 8 & 21 \\
\hline
\end{tabular}

Table 3: The list of selection criteria for category $(\mathrm{C})$. Selection I(a) is specially optimised for the region of $\Delta M_{+} \leq 5 \mathrm{GeV}$. The numbers of background events expected for the integrated luminosity of $57 \mathrm{pb}^{-1}$ for various Standard Model processes and the total number of background events expected as well as the observed number of events in each $\Delta M_{+}$region are also listed. The errors in the total background include only the statistical error of simulated background events.

The net charge of the event must be zero to reject poorly reconstructed events. Since the signal is expected to have a two lepton or two jet topology, the events are split into two jets using the Durham jet algorithm. To ensure that the jet assignment is correct, each jet must 
contain at least a charged track $\left(N_{\mathrm{ch}, \mathrm{j}} \geq 1\right)$, have a significant energy $\left(E_{\mathrm{j}}\right)$ and the magnitude of the sum of the track charges $\left(\left|Q_{\mathrm{j}}\right|\right)$ must not exceed 1. If the acoplanarity angle is small, cuts are applied on the transverse momentum $\left(P_{\mathrm{t}}\right)$, the transverse momentum perpendicular to the event thrust axis $\left(a_{\mathrm{t}}\right)$, and $\left|\cos \theta_{a}\right|\left(\theta_{a} \equiv \tan ^{-1}\left(a_{\mathrm{t}} / P_{z}\right)\right)$. These cuts reduce the background from two-photon processes and lepton pairs. If the acoplanarity angle is large, cuts on $P_{\mathrm{t}}$ and $\left|\cos \theta_{\text {miss }}\right|$ are applied to reduce the two-photon background and reject events that may have particles escaping detection along the beam line. The $P_{\mathrm{t}}$ distributions for the full acoplanarity angle region are shown in Fig. 5 after the cut on the jet charge.

To reduce the background from $\mathrm{e}^{+} \mathrm{e}^{-} \mu^{+} \mu^{-}$events in which one of the muons is emitted at a small polar angle and is not reconstructed as a good track, events are rejected if there is a track segment in the muon chamber, or a hadron calorimeter cluster at a small polar angle, near the missing momentum direction $\left(\vec{P}_{\text {miss }}\right)$ in the plane perpendicular to the beam axis. Soft hadronic events from two-photon processes are rejected by cuts on $\left|\cos \theta_{\mathrm{j}}\right|$, $\phi_{\text {acop }}$ and $M_{\text {vis }}$. $\mathrm{W}^{+} \mathrm{W}^{-} \rightarrow \ell^{+} \nu \ell^{-} \bar{\nu}$ events are rejected by upper cuts on $M_{\mathrm{vis}}$ and on the higher energy of the two jets, $E_{1} / E_{\text {beam. }}$. The distributions of $E_{1} / E_{\text {beam }}$ are shown for region II in Fig. 6 after all the other cuts have been applied. The values and ranges for all cuts are given in Table 3. The typical detection efficiencies for $\tilde{\chi}_{1}^{+} \tilde{\chi}_{1}^{-}$events are shown in Fig. 9a.

The analysis is especially optimised for the case that both charginos decay leptonically into three particles $\left(\tilde{\chi}_{1}^{+} \rightarrow \ell^{+} \nu \tilde{\chi}_{1}^{0}\right)$. In addition the analysis is designed to have high efficiency in the small $\Delta M_{+}$region, where a large fraction of hadronic events fall into category (C). If $m_{\tilde{\nu}}$ is smaller than $m_{\tilde{\chi}_{1}^{+}}$, the two-body decays of the chargino $\left(\tilde{\chi}_{1}^{+} \rightarrow \tilde{\nu} \ell^{+}\right)$may dominate over the three body decays via a virtual $W$. The analysis of Ref. [31] especially tuned for acoplanar lepton search is applied in this case.

\subsection{Detection of Neutralinos}

To obtain optimal performance the event sample is divided exclusively into two categories, motivated by the topologies expected from neutralino events.

(C) $N_{\text {ch }} \leq 4$ : the signal events in which $\tilde{\chi}_{2}^{0}$ decays into $\tilde{\chi}_{1}^{0} \ell^{+} \ell^{-}$tend to fall into this category. When the mass difference between $\tilde{\chi}_{2}^{0}$ and $\tilde{\chi}_{1}^{0}\left(\Delta M_{0} \equiv m_{\tilde{\chi}_{2}^{0}}-m_{\tilde{\chi}_{1}^{0}}\right)$ is small, signal events also tend to fall into this category.

(D) $N_{\mathrm{ch}}>4$ : the signal events in which $\tilde{\chi}_{2}^{0}$ decays into $\tilde{\chi}_{1}^{0} \mathrm{q} \overline{\mathrm{q}}$ tend to fall into this category for modest and large values of $\Delta M_{0}$.

For events with $N_{\mathrm{ch}} \leq 4$ the category (C) cuts of the chargino search are used. Events falling into category $(\mathrm{D})$ have a monojet or di-jet topology and the cuts described below provide better detection-performance for $\tilde{\chi}_{1}^{0} \tilde{\chi}_{2}^{0}$ detection than would have been obtained using the cuts of category (A) of the chargino search.

The fraction of events falling into category (C) is $10-20 \%$ for $\Delta M_{0} \geq 20 \mathrm{GeV}$ but increases to about $70 \%$ when $\Delta M_{0} \leq 5 \mathrm{GeV}$. The fraction of invisible events due to $\tilde{\chi}_{2}^{0} \rightarrow \tilde{\chi}_{1}^{0} \mathrm{Z}^{(*)} \rightarrow \tilde{\chi}_{1}^{0} \nu \bar{\nu}$ decays is $20-30 \%$ depending on $\Delta M_{0}$.

The event shape of $\tilde{\chi}_{1}^{0} \tilde{\chi}_{2}^{0}$ events mainly depends on the difference between the $\tilde{\chi}_{2}^{0}$ mass and the $\tilde{\chi}_{1}^{0}$ mass, therefore the selection criteria are optimised for four $\Delta M_{0}$ regions: 
(i) $\Delta M_{0} \leq 10 \mathrm{GeV}$,

(ii) $10<\Delta M_{0} \leq 30 \mathrm{GeV}$,

(iii) $30<\Delta M_{0} \leq 80 \mathrm{GeV}$,

(iv) $\Delta M_{0}>80 \mathrm{GeV}$.

In regions $\mathrm{i}$ and ii, the main sources of background events are two-photon processes and the $\gamma^{*} Z^{(*)} \rightarrow \mathrm{q} \overline{\mathrm{q}} \nu \bar{\nu}$ processes. In regions iii and iv, the main sources of background are four-fermion processes $\left(\mathrm{W}^{+} \mathrm{W}^{-}\right.$, We $\nu$ and $\left.\gamma^{*} Z^{(*)}\right)$. Selection criteria applied for the low-multiplicity events (category $(\mathrm{C})$ ) for regions i, ii, iii and iv are identical to those used in the analysis (C) of the chargino search for regions Ia, II, III and IV, respectively (see Table 3). The typical efficiencies for detecting $\tilde{\chi}_{1}^{0} \tilde{\chi}_{2}^{0}$ events with the $\tilde{\chi}_{2}^{0} \rightarrow \tilde{\chi}_{1}^{0} \mathrm{Z}^{*}$ decay are shown in Fig. $9 \mathrm{~b}$.

\subsubsection{Analysis (D) $\left(\mathrm{N}_{\mathrm{ch}}>4\right)$}

In $\tilde{\chi}_{1}^{0} \tilde{\chi}_{2}^{0}$ events, if the $\tilde{\chi}_{2}^{0}$ decays hadronically, events tend to fall into category $(\mathrm{D})$. The variables used in the selection criteria and their cut values are listed in Table 1.

To reduce the background from $\mathrm{e}^{+} \mathrm{e}^{-} \rightarrow \mathrm{Z} \gamma$ and two-photon processes, requirements on $\theta_{\text {miss }}$, $E_{\mathrm{fwd}} / E_{\mathrm{vis}}$ and transverse momenta are set. The acoplanarity angle, as defined in the chargino analysis (A), should be large to remove the two-photon and the $q \bar{q}$ background events. To ensure the reliability of the measurement of $\phi_{\text {acop }}$, both jets should have a polar angle $\theta_{\mathrm{j}}$ in the range $\left|\cos \theta_{\mathrm{j}}\right|<0.95$. The acoplanarity angle distribution for region ii after the $\left|\cos \theta_{\mathrm{j}}\right|$ cut is shown in Fig. 7. For region iv the $\phi_{\text {acop }}$ cut is loosened with respect to regions i-iii, since the acoplanarity angle of signal events is smaller. On the other hand the $E_{\mathrm{fwd}} / E_{\mathrm{vis}}$ cut is tightened to reduce the $q \bar{q}$ background.

After these cuts, the remaining background events come predominantly from $\gamma^{*} \mathrm{Z} \rightarrow \mathrm{q} \overline{\mathrm{q}} \nu \bar{\nu}$, $\mathrm{W}^{+} \mathrm{W}^{-} \rightarrow \ell \nu \mathrm{q} \overline{\mathrm{q}}^{\prime}$ and $\mathrm{We} \nu \rightarrow \mathrm{q}^{\prime} \mathrm{e} \nu$. Cuts on the visible mass and on the ratio of the visible mass to the visible energy are then applied to reduce the background from $\gamma^{*} Z \rightarrow q \overline{\mathrm{q}} \nu \bar{\nu}$. In regions iii and iv, $d_{23}^{2} \equiv y_{23} E_{\text {vis }}^{2}<30 \mathrm{GeV}^{2}$ is required to select a clear two-jet topology and to reject $\mathrm{W}^{+} \mathrm{W}^{-} \rightarrow \tau \nu \mathrm{q} \overline{\mathrm{q}}^{\prime}$ events. In Fig.8 the $d_{23}^{2}$ distribution is shown for region iv after all the other cuts. Typical detection efficiencies for $\tilde{\chi}_{2}^{0} \tilde{\chi}_{1}^{0}$ events are shown in Fig. $9 \mathrm{~b}$.

\subsection{Systematic errors and corrections}

Systematic errors on the number of expected signal events arise from the following sources: the measurement of the integrated luminosity (0.5\%), Monte Carlo statistics in the various signal samples, interpolation errors of the efficiencies to arbitrary values of $m_{\tilde{\chi}_{1}^{+}}$and $m_{\tilde{\chi}_{1}^{0}}(2-$ $10 \%$ ), modelling of the cut variables in the Monte Carlo simulations (4-10\%), errors due to fragmentation uncertainties in hadronic decays $(<2 \%)$, the matrix elements leading to different decay parameters $(<5 \%)$ and effects of detector calibration $(<1 \%)$. The effect of possible trigger inefficiencies has been investigated and found to be negligible.

${ }^{4}$ This is estimated by comparing the efficiencies obtained by shifting each cut variable by the possible shift in the corresponding distribution which still gives agreement between data and Monte Carlo. 


\begin{tabular}{|c|c|c|c|c|}
\hline Region & $\mathrm{i}$ & ii & iii & iv \\
\hline 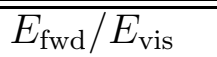 & \multicolumn{2}{|c|}{$<0.15$} & $\overline{<<0.20}$ & $<<0.05$ \\
\hline$\left|\cos \theta_{\text {miss }}\right|$ & \multicolumn{2}{|c|}{$<0.8$} & \multicolumn{2}{|c|}{$<0.9$} \\
\hline$p_{\mathrm{t}} \mathrm{GeV}$ & \multirow{2}{*}{\multicolumn{2}{|c|}{$>5$}} & \multicolumn{2}{|c|}{$>7$} \\
\hline$p_{\mathrm{t}}^{\mathrm{HCAL}} \mathrm{GeV}$ & & & \multicolumn{2}{|c|}{$>7$} \\
\hline$\left|\cos \theta_{\mathrm{j}}\right|$ & \multicolumn{4}{|c|}{$<0.95$} \\
\hline$\phi_{\text {acop }}^{\circ}$ & \multicolumn{3}{|c|}{$>20$} & $>10$ \\
\hline$M_{\text {vis }} \mathrm{GeV}$ & $<12$ & $<35$ & $<70$ & {$[20,130]$} \\
\hline$M_{\mathrm{vis}} / E_{\mathrm{vis}}$ & \multicolumn{3}{|c|}{$>0.3$} & - \\
\hline$d_{23}^{2} \mathrm{GeV}^{2}$ & \multicolumn{2}{|c|}{-} & \multicolumn{2}{|c|}{$<30$} \\
\hline \multicolumn{5}{|l|}{ background } \\
\hline$\gamma \gamma$ & 1.05 & 2.15 & 0.77 & 0.00 \\
\hline$\ell^{+} \ell^{-}(\gamma)$ & 0.00 & 0.03 & 0.07 & 0.10 \\
\hline $\mathrm{q} \overline{\mathrm{q}}(\gamma)$ & 0.01 & 0.01 & 0.04 & 0.07 \\
\hline $4 \mathrm{f}$ & 0.12 & 1.29 & 3.18 & 10.37 \\
\hline total bkg & $1.19 \pm 0.50$ & $3.49 \pm 0.77$ & 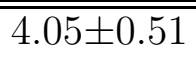 & $10.55 \pm 0.35$ \\
\hline observed & 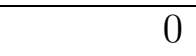 & $\overline{11}$ & 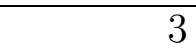 & 12 \\
\hline
\end{tabular}

Table 4: The list of selection criteria for $\tilde{\chi}_{2}^{0} \tilde{\chi}_{1}^{0}$ events in category (D). The numbers of background events expected for the integrated luminosity of $57 \mathrm{pb}^{-1}$ for various Standard Model processes and the total number of background events expected as well as the observed number of events in each $\Delta M_{0}$ region are also listed. The errors in the total background include only the statistical error of simulated background events.

Systematic errors on the expected number of background events are due to Monte Carlo statistics in the simulated background events (as quoted in Tables 1, 2, 3 and 1), uncertainties in the amount of two-photon background which are estimated by fitting the $P_{t}$ distributions of simulated two-photon events and the data (30\%), and uncertainties in the simulation of the four-fermion processes which are estimated by taking the difference between the predictions of the grc4f [21] and the EXCALIBUR [32] generators (17\%). The systematic errors due to the modelling of the cut variables in the detector simulation are less than $7 \%$.

The rate of events in which the measured energy in the SW, FD or GC calorimeters, due to noise and beam related background, exceeded the thresholds in the preselection is $4.5 \%$ as estimated from random beam crossing events. Since this effect is not modelled in the simulation, it is taken into account by scaling down the integrated luminosity by this amount.

\section{Results}

\subsection{Limits on the $\tilde{\chi}_{1}^{+} \tilde{\chi}_{1}^{-}$and $\tilde{\chi}_{2}^{0} \tilde{\chi}_{1}^{0}$ production cross-sections}

Model-independent upper limits are obtained at 95\% C.L. on the production cross-sections. This is done for $\tilde{\chi}_{1}^{+} \tilde{\chi}_{1}^{-}$assuming the specific decay mode $\tilde{\chi}_{1}^{ \pm} \rightarrow \tilde{\chi}_{1}^{0} \mathrm{~W}^{(*) \pm}$ and for $\tilde{\chi}_{1}^{0} \tilde{\chi}_{2}^{0}$ production assuming the $\tilde{\chi}_{2}^{0} \rightarrow \tilde{\chi}_{1}^{0} Z^{(*)}$ decay. Exclusion regions are determined from the observed numbers 
of events at $\sqrt{s}=181-184 \mathrm{GeV}$, the signal detection efficiencies and their uncertainties, and the numbers of background events expected and their uncertainties. To obtain this limit at a given $\left(m_{\tilde{\chi}_{1}^{+}}, m_{\tilde{\chi}_{1}^{0}}\right)$ or $\left(m_{\tilde{\chi}_{2}^{0}}, m_{\tilde{\chi}_{1}^{0}}\right)$ point, the independent analyses $((\mathrm{A}),(\mathrm{B})$ and $(\mathrm{C})$ for chargino, (C) and (D) for neutralino) are combined using the likelihood ratio method [33]. This method assigns greater weight to the analysis which has greater sensitivity.

Systematic uncertainties on the efficiencies are incorporated following the method in Ref. [34, and the systematic uncertainties on the number of expected background events are incorporated by numerical integration, assuming Gaussian errors, as suggested in Ref. [34].

Contours of the $95 \%$ C.L. upper limits for the $\tilde{\chi}_{1}^{+} \tilde{\chi}_{1}^{-}$cross-sections are shown in Fig. 10 assuming the $\tilde{\chi}_{1}^{ \pm} \rightarrow \tilde{\chi}_{1}^{0} \mathrm{~W}^{(*) \pm}$ decay with $100 \%$ branching fraction. Although these limits do not depend on the details of the SUSY models considered, a "typical" field content of the gauginos is assumed, leading to particular production angular distributions that are subsequently used in estimating detection efficiencies. Differences in detection efficiencies arise from variations in the angular distributions obtained by using different MSSM parameters corresponding to the same mass combination. The variation of the efficiency is observed to be less than $2 \%$. Of the parameters examined, those yielding the lowest efficiency are used. If the cross-section for $\tilde{\chi}_{1}^{+} \tilde{\chi}_{1}^{-}$is larger than $0.6 \mathrm{pb}$ and $\Delta M_{+}$is between $5 \mathrm{GeV}$ and about $80 \mathrm{GeV}, m_{\tilde{\chi}_{1}^{+}}$is excluded with $95 \%$ C.L. up to the kinematic limit, assuming $\operatorname{Br}\left(\tilde{\chi}_{1}^{+} \rightarrow \tilde{\chi}_{1}^{0} \mathrm{~W}^{(*)+}\right)=100 \%$.

Similar contours of the upper limits for the $\tilde{\chi}_{2}^{0} \tilde{\chi}_{1}^{0}$ cross-sections are shown in Fig. 11. If the cross-section for $\tilde{\chi}_{2}^{0} \tilde{\chi}_{1}^{0}$ is larger than $0.3 \mathrm{pb}$ and $\Delta M_{0}$ is greater than $10 \mathrm{GeV}, m_{\tilde{\chi}_{2}^{0}}$ is excluded up to the kinematic limit at $95 \%$ C.L., assuming $\operatorname{Br}\left(\tilde{\chi}_{2}^{0} \rightarrow \tilde{\chi}_{1}^{0} Z^{(*)}\right)=100 \%$.

\subsection{Limits in the MSSM parameter space}

The results of the above searches can be interpreted within the framework of the Constrained MSSM (CMSSM). The phenomenology of the gaugino-higgsino sector of the MSSM is mostly determined by the parameters $M_{2}, \mu$ and $\tan \beta$. In the absence of light sfermions and light SUSY Higgs particles, these three parameters are sufficient to describe the chargino and neutralino sectors completely. Within the CMSSM, a large value of the common scalar mass, $m_{0}$ (e.g., $m_{0}=500 \mathrm{GeV}$ ) leads to heavy sfermions and therefore to a negligible suppression of the crosssection due to interference from $t$-channel sneutrino exchange. Chargino decays would then proceed predominantly via a virtual or real $W$. On the other hand, a light $m_{0}$ results in a low value of the mass of the $\tilde{\nu}$, enhancing the contribution of the $t$-channel exchange diagrams that may have destructive interference with $s$-channel diagrams, thus reducing the cross-section for chargino pair production. Small values of $m_{0}$ also tend to enhance the leptonic branching ratio of charginos, often leading to smaller detection efficiencies. Certain values of $m_{0}$ can lead to the condition $m_{\tilde{\nu}}<m_{\tilde{\chi}_{1}^{ \pm}}$and result in the two-body decay mode $\tilde{\chi}_{1}^{ \pm} \rightarrow \tilde{\nu} \ell^{ \pm}$being dominant. The chargino detection efficiency can be small or zero for these decays, particularly when $m_{\tilde{\nu}} \approx m_{\tilde{\chi}_{1}^{ \pm}}$, leading to severe degradation in sensitivity.

From the input parameters $M_{2}, \mu, \tan \beta, m_{0}$ and $A$ (the trilinear coupling), masses, production cross-sections and branching fractions are calculated according to the CMSSM [8, 9, 10, 12].

${ }^{5}$ When calculating limits, cross-sections at different $\sqrt{s}$ are estimated by weighting by $\bar{\beta} / s$ in these proportions, where $\bar{\beta}$ is $p_{\tilde{\chi}_{1}^{ \pm}} / E_{\text {beam }}$ for $\tilde{\chi}_{1}^{+} \tilde{\chi}_{1}^{-}$production or $p_{\tilde{\chi}_{2}^{0}} / E_{\text {beam }}=p_{\tilde{\chi}_{1}^{0}} / E_{\text {beam }}$ for $\tilde{\chi}_{2}^{0} \tilde{\chi}_{1}^{0}$ production. 
For each set of input parameters, the total number of $\tilde{\chi}_{1}^{+} \tilde{\chi}_{1}^{-}, \tilde{\chi}_{2}^{0} \tilde{\chi}_{1}^{0}, \tilde{\chi}_{3}^{0} \tilde{\chi}_{1}^{0}$ and $\tilde{\chi}_{2}^{0} \tilde{\chi}_{2}^{0}$ events expected to be observed are calculated using the integrated luminosity, the cross-sections, branching fractions, and the detection efficiencies which depend upon the masses of the chargino, the lightest neutralino and next-to-lightest neutralino. The relative importance of each of the analyses (A)-(D) changes with the leptonic or hadronic branching ratios, and the likelihood ratio method [33] is used to optimally weight each analysis depending on these branching ratios.

Results are presented for two cases: (i) $m_{0}=500 \mathrm{GeV}$ (i.e., heavy sfermions), and (ii) the value of $m_{0}$ that gives the smallest total number of expected chargino and neutralino events taking into account cross-sections, branching ratios, and detection efficiencies for each set of values of $M_{2}, \mu, \tan \beta$. This value of $m_{0}$ hence leads to the worst limit at that point, so that the resulting limits are valid for all $m_{0}$. Values of $m_{0}$ are considered that remain compatible with the current limits on the $\tilde{\nu}$ mass $\left(m_{\tilde{\nu}_{L}}>43 \mathrm{GeV}\right.$ [35]), and OPAL upper limits on the cross-section for $\tilde{\ell}$ pair production, particularly right-handed smuon and selectron pair production [31. Particular attention is paid to the region of values of $m_{0}$ leading to the critical mass condition $m_{\tilde{\nu}} \approx m_{\tilde{\chi}_{1}^{ \pm}}$. When $m_{\tilde{\nu}} \leq m_{\tilde{\chi}_{1}^{ \pm}}$, resulting in a topology of acoplanar leptons and missing momentum, the upper limits on the cross-section for the two-body chargino decay from Ref. [31] are used. The contribution of the cascade decays $\tilde{\chi}_{1}^{+} \rightarrow \tilde{\chi}_{2}^{0} \mathrm{X}$ followed by $\tilde{\chi}_{2}^{0} \rightarrow \tilde{\chi}_{1}^{0} \mathrm{Y}$ are also included. The photonic radiative decay $\tilde{\chi}_{2}^{0} \rightarrow \tilde{\chi}_{1}^{0} \gamma$ leading to single photon topologies from $\tilde{\chi}_{2}^{0} \tilde{\chi}_{1}^{0}$ production and acoplanar photons with missing energy topologies from $\tilde{\chi}_{2}^{0} \tilde{\chi}_{2}^{0}$ are taken into account using the $95 \%$ C.L. cross-section upper limits on these topologies from the OPAL results of Ref. [13]. In both of these cases, if the relevant product of cross-section and branching ratio for a particular set of MSSM parameters is greater than the measured 95\% C.L. upper limit presented in that paper, then that set of parameters is considered to be excluded.

The following regions of the CMSSM parameters are scanned: $0 \leq M_{2} \leq 2000 \mathrm{GeV}$, $|\mu| \leq 500 \mathrm{GeV}$, and $A= \pm M_{2}, \pm m_{0}$ and 0 . The typical scan step is $0.2 \mathrm{GeV}$. Checks have been made to ensure that the scanned ranges of parameters are large enough that the exclusion regions change negligibly for even larger ranges. No significant dependence on $A$ is observed. The $95 \%$ C.L. upper limit on the expected number of events is determined and systematic errors on efficiencies and backgrounds are incorporated as described previously. Figure 12 shows the resulting exclusion regions in the $\left(M_{2}, \mu\right)$ plane for $\tan \beta=1.5$ and 35 with $m_{0} \geq 500 \mathrm{GeV}$ and for all $m_{0}$.

The restrictions on the CMSSM parameter space presented here can be transformed into exclusion regions in the $\left(m_{\tilde{\chi}_{1}^{ \pm}}, m_{\tilde{\chi}_{1}^{0}}\right)$ or $\left(m_{\tilde{\chi}_{2}^{0}}, m_{\tilde{\chi}_{1}^{0}}\right)$ plane. A given mass pair is excluded only if all CMSSM parameters in the scan which lead to that same mass pair are excluded at the 95\% C.L. The $\tilde{\chi}_{1}^{ \pm}$mass limits are summarised in Table 5 . In the $\left(m_{\tilde{\chi}_{1}^{ \pm}}, m_{\tilde{\chi}_{1}^{0}}\right)$ plane, Fig. 13 shows the corresponding $95 \%$ C.L. exclusion regions for $\tan \beta=1.5$ and 35 .

Figure 14 shows the corresponding $95 \%$ C.L. exclusion regions in the $\left(m_{\tilde{\chi}_{2}^{0}}, m_{\tilde{\chi}_{1}^{0}}\right)$ plane, for $\tan \beta=1.5$ and 35. Although the neutralino production cross-section is small, our detection efficiencies in the direct neutralino search are high enough, and we have now collected enough integrated luminosity that, for certain SUSY parameters, we should be able to observe them directly rather than their being excluded indirectly through the exclusion of charginos of certain masses. Regions that would be excluded by the direct neutralino searches alone are also shown in Fig. 14 delimited by the white dotted lines. Mass limits on $\tilde{\chi}_{1}^{0}, \tilde{\chi}_{2}^{0}$, and $\tilde{\chi}_{3}^{0}$ are summarised in Table 6 .

To study the sensitivity of these numerical mass limits, the limits expected from the Standard Model background processes are computed assuming no signal. For chargino mass limits 


\begin{tabular}{|c|c||c|c|}
\hline & & $\tan \beta=1.5$ & $\tan \beta=35$ \\
\hline \hline$m_{0} \geq 500 \mathrm{GeV}$ & $\Delta M_{+} \geq 5 \mathrm{GeV}$ & $m_{\tilde{\chi}_{1}^{+}}>90.0 \mathrm{GeV}$ & $m_{\tilde{\chi}_{1}^{+}}>90.2 \mathrm{GeV}$ \\
& $\Delta M_{+} \geq 10 \mathrm{GeV}$ & $m_{\tilde{\chi}_{1}^{+}}>91.1 \mathrm{GeV}$ & $m_{\tilde{\chi}_{1}^{+}}>91.2 \mathrm{GeV}$ \\
\hline All $m_{0}$ (see text) & $\Delta M_{+} \geq 5 \mathrm{GeV}$ & $m_{\tilde{\chi}_{1}^{+}}>69.1 \mathrm{GeV}$ & $m_{\tilde{\chi}_{1}^{+}}>65.2 \mathrm{GeV}$ \\
\hline
\end{tabular}

Table 5: Lower limits at 95\% C.L. obtained on the lightest chargino mass.

\begin{tabular}{|c|c||c|c|}
\hline & & $\tan \beta=1.5$ & $\tan \beta=35$ \\
\hline \hline$m_{0} \geq 500 \mathrm{GeV}$ & No $\Delta M_{0}$ restriction & $m_{\tilde{\chi}_{1}^{0}}>38.1 \mathrm{GeV}$ & $m_{\tilde{\chi}_{1}^{0}}>46.4 \mathrm{GeV}$ \\
\cline { 2 - 4 } & $\Delta M_{0} \geq 10 \mathrm{GeV}$ & $m_{\tilde{\chi}_{2}^{0}}>63.0 \mathrm{GeV}$ & $m_{\tilde{\chi}_{2}^{0}}>91.1 \mathrm{GeV}$ \\
& & $m_{\tilde{\chi}_{3}^{0}}>102.3 \mathrm{GeV}$ & $m_{\tilde{\chi}_{3}^{0}}>122.1 \mathrm{GeV}$ \\
\hline All $m_{0}$ (see text) & No $\Delta M_{0}$ restriction & $m_{\tilde{\chi}_{1}^{0}}>25.4 \mathrm{GeV}$ & $m_{\tilde{\chi}_{1}^{0}}>36.5 \mathrm{GeV}$ \\
\cline { 2 - 4 } & $\Delta M_{0} \geq 10 \mathrm{GeV}$ & $m_{\tilde{\chi}_{2}^{0}}>51.7 \mathrm{GeV}$ & $m_{\tilde{\chi}_{2}^{0}}>84.8 \mathrm{GeV}$ \\
& & $m_{\tilde{\chi}_{3}^{0}}>102.1 \mathrm{GeV}$ & $m_{\tilde{\chi}_{3}^{0}}>124.4 \mathrm{GeV}$ \\
\hline
\end{tabular}

Table 6: Lower limits at $95 \%$ C.L. obtained on $m_{\tilde{\chi}_{1}^{0}}, m_{\tilde{\chi}_{2}^{0}}$, and $m_{\tilde{\chi}_{3}^{0}}$.

close to the kinematic limit, the expected limits differ by less than $0.2 \mathrm{GeV}$ from the observed limit values, while expected mass limits for other values in Table 5 and Table 6 differ by less than $3.0 \mathrm{GeV}$ from the observed values in all cases.

In the "Higgsino region" where $M_{2}$ is large, the mass difference $\Delta M_{+}$between the chargino and lightest neutralino decreases with increasing $M_{2}$, resulting in a drop in detection efficiency. For $\tan \beta=1.5$, Fig. 15 illustrates the gaugino mass limits in slices of constant $M_{2}$ as well as the correspondence of $M_{2}$ with mass difference $\Delta M_{+}$. For $\mu<0$ the $m_{\tilde{\chi}_{1}^{+}}$limit is above the kinematical boundary of $\tilde{\chi}_{1}^{+} \tilde{\chi}_{1}^{-}$production. This is obtained from the interpretation of the results of the direct $\tilde{\chi}_{2}^{0} \tilde{\chi}_{1}^{0}$ searches. For larger values of $\tan \beta$ the form of the curves are similar, but the difference between the limits for $\mu<0$ and $\mu>0$ for a particular gaugino decreases, and the limits are in general between the $\mu<0$ and $\mu>0$ curves shown for $\tan \beta=1.5$.

Figure 16 illustrates the sensitivity of the chargino mass limit to the mass of the sneutrino set by the chosen value of $m_{0}$. The dashed line delineates the condition $m_{\tilde{\chi}_{1}^{ \pm}}=m_{\tilde{\nu}}$ where the $\tilde{\chi}_{1}^{+} \tilde{\chi}_{1}^{-}$search fails due to small visible energy. In the region to the right side of the line, charginos will undergo three-body decays which are searched for directly, while on the left side of the line they decay via the two-body mode $\tilde{\chi}^{ \pm} \rightarrow \tilde{\nu} \ell^{ \pm}$and the results of Ref. [31] are applied. In this case the chargino lower mass limit is set mostly by the right-handed slepton limits with some contributions from neutralino production.

Figure 17 shows the dependence of the mass limits on the value of $\tan \beta$. Of particular interest is the absolute lower limit, in the framework of the CMSSM, on the mass of the lightest neutralino of $m_{\tilde{\chi}_{1}^{0}}>30.1 \mathrm{GeV}(24.2 \mathrm{GeV})$ at $95 \%$ C.L. for $m_{0} \geq 500 \mathrm{GeV}$ (all $\left.m_{0}\right)$. This has implications on direct searches for the lightest neutralino as a candidate for dark matter. If the lightest neutralino forms dark matter and it is lighter than about $20 \mathrm{GeV}$, it is difficult to detect it by terrestrial dark matter searches due to the small recoil energy in the material [36]. Since the formulae for couplings and masses in the gaugino sector are symmetric in $\tan \beta$ and $1 / \tan \beta$, these results also hold for $\tan \beta<1$. 


\section{Summary and Conclusion}

A data sample corresponding to an integrated luminosity of $57 \mathrm{pb}^{-1}$ at $\sqrt{s}=181-184 \mathrm{GeV}$, collected with the OPAL detector, has been analysed to search for pair production of charginos and neutralinos predicted by supersymmetric theories. No evidence for these events has been observed. Assuming $m_{\tilde{\chi}_{1}^{ \pm}}-m_{\tilde{\chi}_{1}^{0}} \geq 5 \mathrm{GeV}$, the $95 \%$ C.L. lower mass limit of the chargino is $90.0 \mathrm{GeV}$ for $\tan \beta=1.5$ and $90.2 \mathrm{GeV}$ for $\tan \beta=35$, within the framework of the CMSSM and for the case of a large universal scalar mass $\left(m_{0} \geq 500 \mathrm{GeV}\right)$. For all $m_{0}$, the 95\% C.L. lower mass limit is $69.1 \mathrm{GeV}$ for $\tan \beta=1.5$ and $65.2 \mathrm{GeV}$ for $\tan \beta=35$. In certain regions of

parameter space, the $m_{\tilde{\chi}_{1}^{ \pm}}$limit exceeds the kinematical boundary of the $\tilde{\chi}_{1}^{+} \tilde{\chi}_{1}^{-}$production due to the interpretation of the results from the direct $\tilde{\chi}_{2}^{0} \tilde{\chi}_{1}^{0}$ search. The absolute lower mass limit on the lightest neutralino in the framework of the CMSSM with $m_{0} \geq 500 \mathrm{GeV}$ is $30.1 \mathrm{GeV}$ at $95 \%$ C.L., and $24.2 \mathrm{GeV}$ for the worst-case limit with all $m_{0}$. This has implications for experimental searches for the lightest neutralino as a dark matter candidate.

\section{Acknowledgements}

We particularly wish to thank the SL Division for the efficient operation of the LEP accelerator at all energies and for their continuing close cooperation with our experimental group. We thank our colleagues from CEA, DAPNIA/SPP, CE-Saclay for their efforts over the years on the time-of-flight and trigger systems which we continue to use. In addition to the support staff at our own institutions we are pleased to acknowledge the

Department of Energy, USA, National Science Foundation, USA, Particle Physics and Astronomy Research Council, UK, Natural Sciences and Engineering Research Council, Canada, Israel Science Foundation, administered by the Israel Academy of Science and Humanities, Minerva Gesellschaft, Benoziyo Center for High Energy Physics,

Japanese Ministry of Education, Science and Culture (the Monbusho) and a grant under the Monbusho International Science Research Program, Japanese Society for the Promotion of Science (JSPS), German Israeli Bi-national Science Foundation (GIF), Bundesministerium für Bildung, Wissenschaft, Forschung und Technologie, Germany, National Research Council of Canada, Research Corporation, USA, Hungarian Foundation for Scientific Research, OTKA T-016660, T023793 and OTKA F-023259.

\section{References}

[1] Y. Gol'fand and E. Likhtam, JETP Lett. 13 (1971) 323;

D. Volkov and V. Akulov, Phys. Lett. B46 (1973) 109;

J. Wess and B. Zumino, Nucl. Phys. B70 (1974) 39. 
[2] OPAL Collab., K. Ackerstaff et al., Euro. Phys. J. C2 (1998) 213.

[3] OPAL Collab., G. Alexander et al., Phys. Lett. B377 (1996) 181.

[4] OPAL Collab., K. Ackerstaff et al., Phys. Lett. B389 (1996) 616.

[5] ALEPH Collab., D. Buskulic et al., Phys. Lett. B373 (1996) 246; DELPHI Collab., P. Abreu et al., Phys. Lett. B382 (1996) 323;

L3 Collab., M. Acciarri et al., Phys. Lett. B377 (1996) 289.

[6] ALEPH Collab., R. Barate et al., Euro. Phys. J. C2 (1998) 417;

DELPHI Collab., P. Abreu et al., Euro. Phys. J. C1 (1998) 1;

L3 Collab., M. Acciarri et al., CERN preprint CERN-PPE/97-130 (1997).

[7] P. Fayet, ' Unification of the Fundamental Particle Interactions', Plenum Press (1980) 587.

[8] A. Bartl, H. Fraas and W. Majerotto, Z. Phys. C30 (1986) 441;

A. Bartl, H. Fraas and W. Majerotto, Z. Phys. C41 (1988) 475;

A. Bartl, H. Fraas, W. Majerotto and B. Mösslacher, Z. Phys. C55 (1992) 257.

[9] M. Chen, C. Dionisi, M. Martinez and X. Tata, Phys. Rep. 159 (1988) 201;

J. L. Feng and M. J. Strassler, Phys. Rev. D51 (1995) 4661.

[10] A. Bartl, H. Fraas and W. Majerotto, Nucl. Phys. B278 (1986) 1;

S. Ambrosanio and B. Mele, Phys. Rev. D52 (1995) 3900.

[11] H. Haber and D. Wyler, Nucl. Phys. B323 (1989) 267;

H. Komatsu and J. Kubo, Phys. Lett. B157 (1985) 90 and B162 (1985) 379.

[12] M. Carena, J.R. Espinosa, M. Quiros and C.E.M. Wagner, Phys. Lett. B355 (1995) 209.

[13] OPAL Collab., K. Ackerstaff et al., CERN preprint CERN-PPE/97-132, Eur. Phys. Journal C2 (1998) 607.

[14] OPAL Collab., K. Ahmet et al., Nucl. Instr. Meth. A305 (1991) 275;

S. Anderson et al., Nucl. Instr. Meth. A403 (1998) 326;

B.E. Anderson et al., IEEE Trans. on Nucl. Science 41 (1994) 845.

[15] C. Dionisi et al., in 'Physics at LEP2', eds. G. Altarelli, T. Sjöstrand and F. Zwirner, CERN 96-01, vol.2 (1996) 337.

[16] T. Sjöstrand, Comp. Phys. Comm. 82 (1994) 74;

T. Sjöstrand, Lund University report LU TP 95-20.

[17] E. Accomando et al., 'Event Generators for Discovery Physics', hep-ph/9602203, Feb. 1996, and in 'Physics at LEP2', eds. G. Altarelli, T. Sjöstrand and F. Zwirner, CERN 96-01, vol.2 (1996) 299.

[18] E. Boudinov et al., ' $\gamma \gamma$ Event Generators', hep-ph/9512371, Dec. 1995, and in 'Physics at LEP2', eds. G. Altarelli, T. Sjöstrand and F. Zwirner, CERN 96-01, vol.2 (1996) 187.

[19] G. Marchesini et al., Comp. Phys. Comm. 67 (1992) 465. 
[20] J.A.M. Vermaseren, Nucl. Phys. B229 (1983) 347.

[21] J. Fujimoto et al., Comp. Phys. Comm. 100 (1997) 128.

[22] S. Jadach, B.F.L. Ward and Z. Wạs, Comp. Phys. Comm. 79 (1994) 503.

[23] S. Jadach, W. Płaczek and B.F.L. Ward, in 'Physics at LEP2', eds. G. Altarelli, T. Sjöstrand and F. Zwirner, CERN 96-01, vol. 2 (1996) 286.

[24] J. Allison et al., Nucl. Instr. Meth. A317 (1992) 47.

[25] OPAL Collab., R. Akers et al., Phys. Lett. B327 (1994) 411.

[26] OPAL Collab., R. Akers et al., Z. Phys. C65 (1994) 17.

[27] OPAL Collab., P. Acton et al., Z. Phys. C58 (1993) 523.

[28] OPAL Collab., R. Akers et al., Z. Phys. C60 (1993) 199.

[29] OPAL Collab., G. Alexander et al., Z. Phys. C52 (1991) 175.

[30] R.M. Barnett et al. (Particle Data Group), Phys. Rev. D54 (1996) Eq. (28.40).

[31] OPAL Collab., K. Ackerstaff et al., CERN-EP/98-122, submitted to Euro. Phys. J. C.

[32] F.A. Berends, R. Pittau and R. Kleiss, Comp. Phys. Comm. 85 (1995) 437.

[33] A.G. Frodesen, O. Skeggestad, and H. Tofte, 'Probability and Statistics in Particle Physics', Universitetsforlaget, 1979, ISBN 82-00-01-01906-3;

S.L. Meyer, 'Data Analysis for Scientists and Engineers', John Wiley and Sons, 1975, ISBN 0-471-59995-6.

[34] R.D. Cousins and V.L. Highland, Nucl. Instr. Meth. A320 (1992) 331.

[35] C. Caso et al. (Particle Data Group), Euro. Phys. J. C3 (1998) 1.

[36] G.G. Raffelt, 'Dark Matter: Motivation, Candidates and Search', in Proceedings of the 1997 European school of High-Energy Physics, e-print hep-ph/9712538;

H.V. Klapdor-Kleingrothaus and A. Staudt, 'Non-accelerator Particle Physics', IOP Publishing Ltd. (1995) pp. 384-416;

W. Ootani et al., preprint RESCEU-35/97, e-print hep-ex/9709019. 


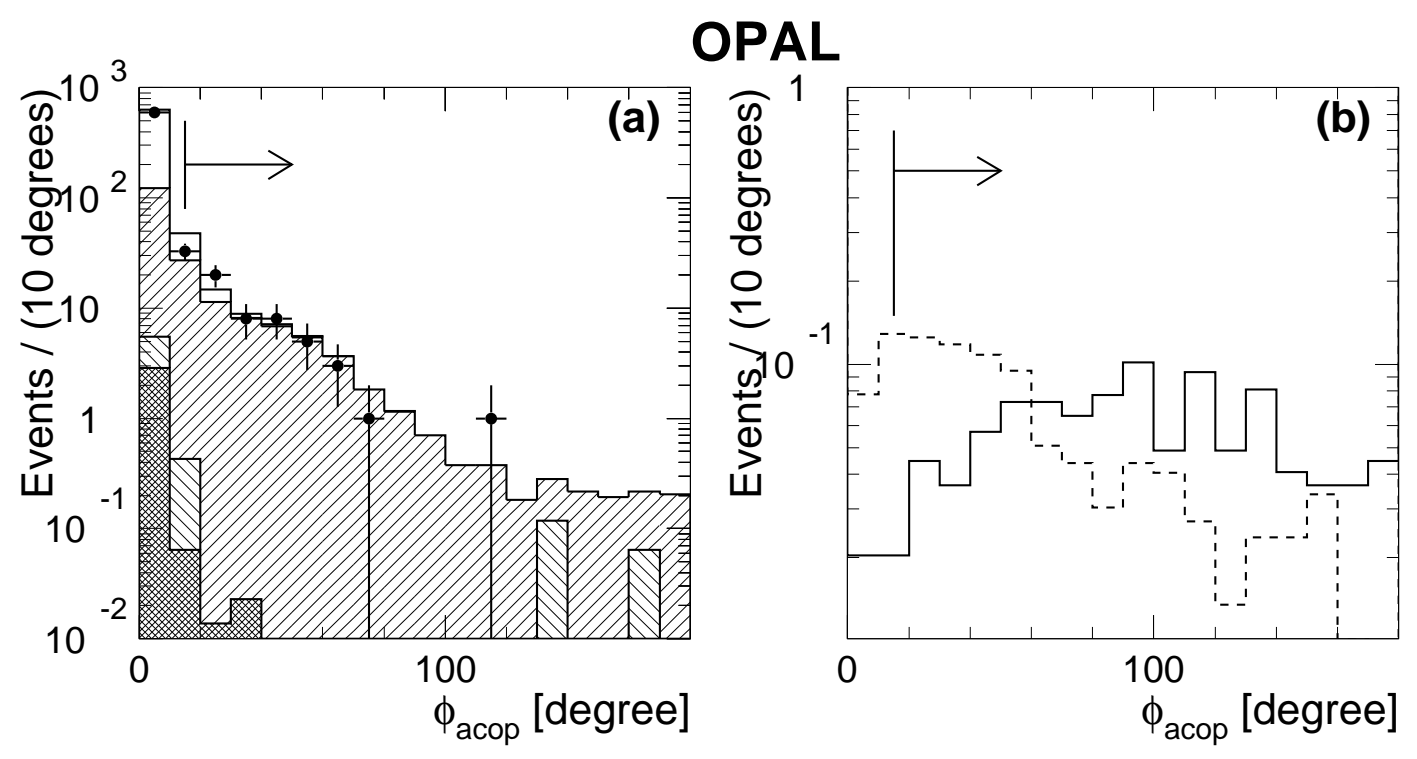

Figure 1: The distributions of the acoplanarity angle, $\phi_{\text {acop }}$, in analysis (A) region II. In (a) are shown the data distribution (dark circles) and the predicted contributions from background processes: dilepton events (double hatched area), two-photon processes (negative slope hatched area), four-fermion processes (including W-pair events) (positive slope hatched area), and multihadronic events (open area). In each case the distribution has been normalised to $57 \mathrm{pb}^{-1}$. In (b) predictions from simulated chargino events are shown for $m_{\tilde{\chi}_{1}^{ \pm}}=90 \mathrm{GeV}$ and $m_{\tilde{\chi}_{1}^{0}}=70 \mathrm{GeV}$ (solid line histogram) and for $m_{\tilde{\chi}_{1}^{ \pm}}=90 \mathrm{GeV}$ and $m_{\tilde{\chi}_{1}^{0}}=45 \mathrm{GeV}$ (dashed line histogram). The normalisations of the signal distributions are arbitrary. The arrows shown indicate the selection criteria.

\section{OPAL}
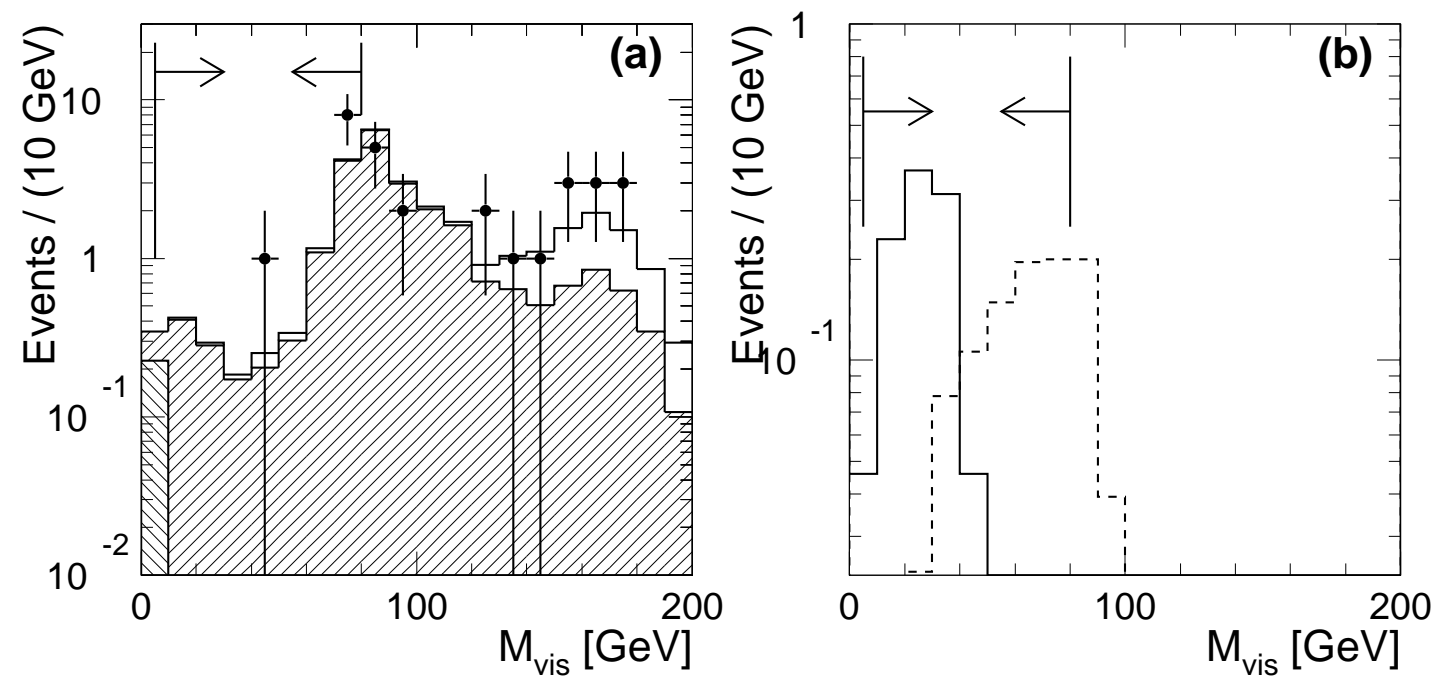

Figure 2: The distributions of $M_{\text {vis }}$ in analysis (A) for data and simulated background events are shown in (a) after the $\phi_{\text {acop }}$ cut for region II. The background sources are shaded as in Fig.1. Distributions of chargino signal events are shown in (b) for $m_{\tilde{\chi}_{1}^{ \pm}}=90 \mathrm{GeV}$ and $m_{\tilde{\chi}_{1}^{0}}=70 \mathrm{GeV}$ (solid line) and for $m_{\tilde{\chi}_{1}^{ \pm}}=90 \mathrm{GeV}$ and $m_{\tilde{\chi}_{1}^{0}}=45 \mathrm{GeV}$ (dashed line). The normalisations of the signal distributions are arbitrary. The arrows shown indicate the selection criteria. 


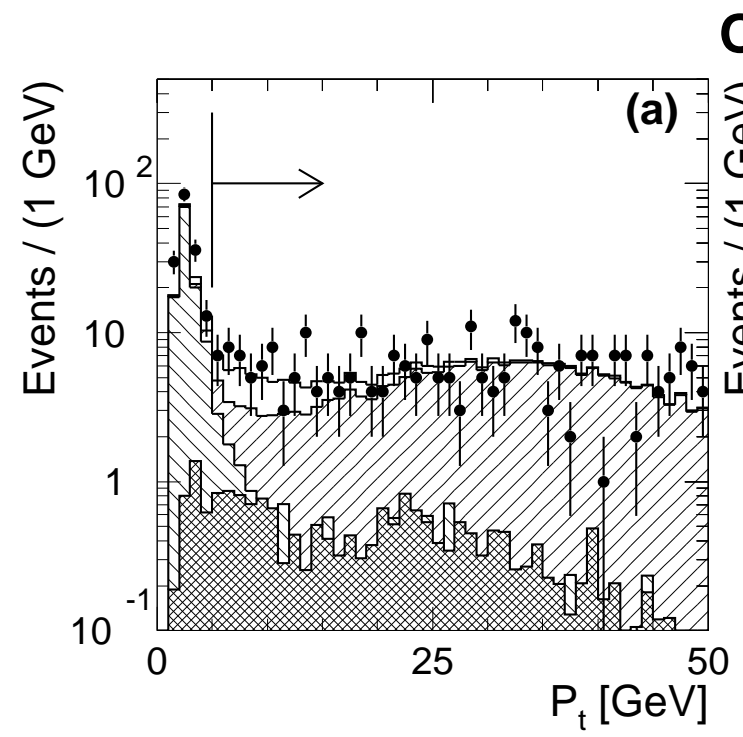

OPAL

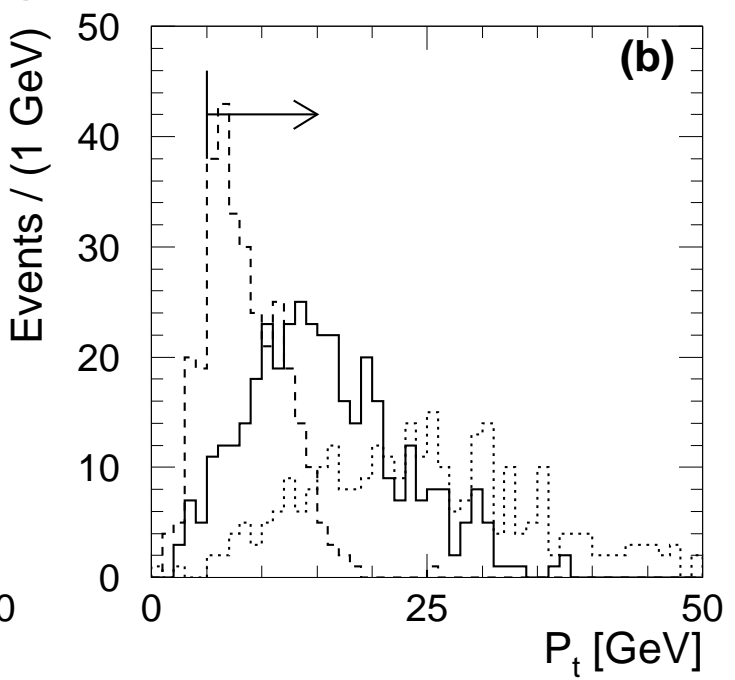

Figure 3: The distributions of $P_{t}$ in analysis (B) region II after the $\left|\cos \theta_{\text {miss }}\right|$ cut. Data and expected background contributions are shown in (a). The background sources are shaded as in Fig.1. The distributions of the signal for simulated chargino events with $m_{\tilde{\chi}_{1}^{+}}=90 \mathrm{GeV}$ and $m_{\tilde{\chi}_{1}^{0}}=70 \mathrm{GeV}$ (solid histogram), with $m_{\tilde{\chi}_{1}^{+}}=90 \mathrm{GeV}$ and $m_{\tilde{\chi}_{1}^{0}}=80 \mathrm{GeV}$ (dashed histogram) and with $m_{\tilde{\chi}_{1}^{+}}=90 \mathrm{GeV}$ and $m_{\tilde{\chi}_{1}^{0}}=45 \mathrm{GeV}$ (dotted histogram) are shown in (b). The normalisations of the signal distributions are arbitrary. The arrows shown indicate the selection criteria.

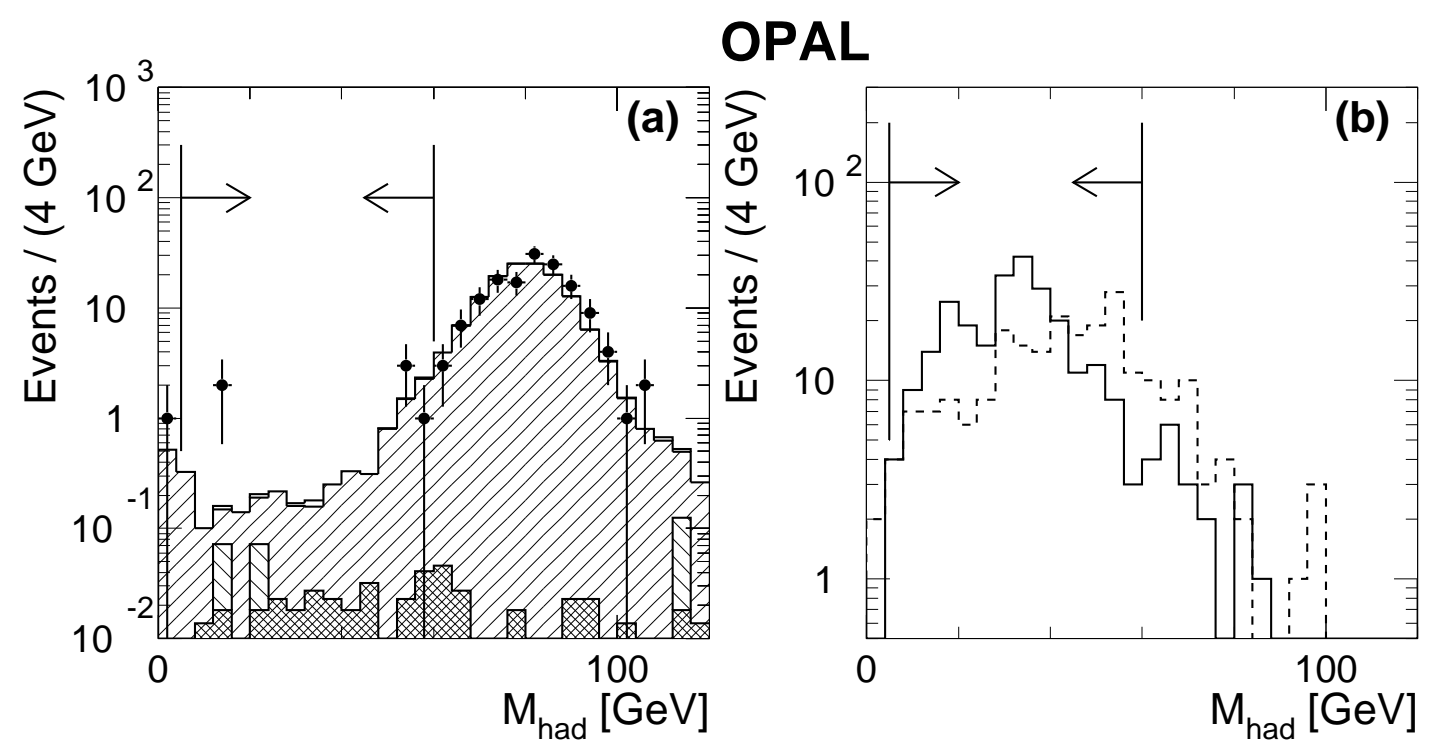

Figure 4: $\quad M_{\text {had }}$ distributions in analysis (B) region III after the $\phi_{\text {acop }}$ cut. Figure (a) shows the data and the Monte Carlo prediction for background processes. The background sources are shaded as in Fig. 1. The distributions of the signal for simulated chargino events with $m_{\tilde{\chi}_{1}^{+}}=90 \mathrm{GeV}$ and $m_{\tilde{\chi}_{1}^{0}}=45 \mathrm{GeV}$ (solid histogram) and with $m_{\tilde{\chi}_{1}^{+}}=90 \mathrm{GeV}$ and $m_{\tilde{\chi}_{1}^{0}}=20 \mathrm{GeV}$ (dashed histogram) are shown in (b). The normalisations of the signal distributions are arbitrary. The arrows shown indicate the selection criteria. 


\section{OPAL}
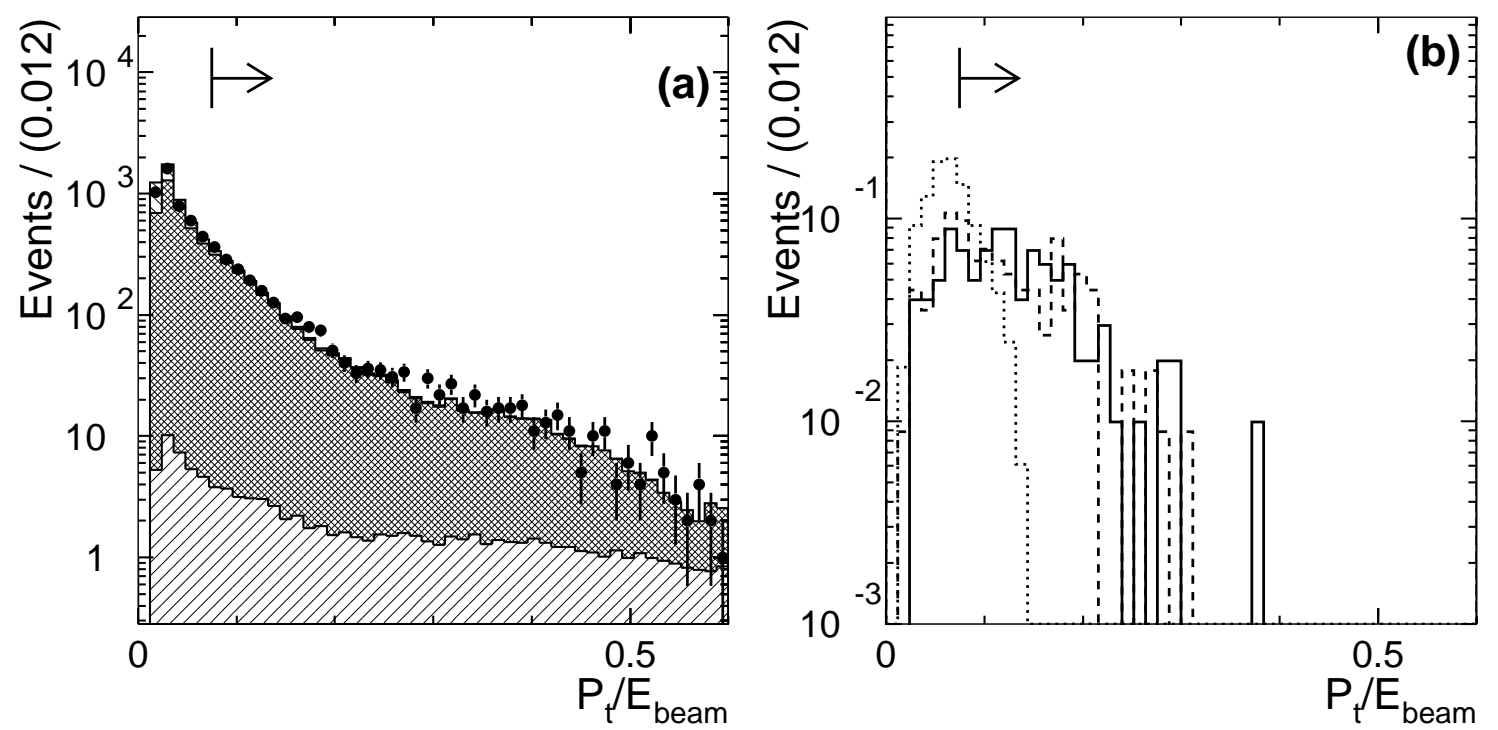

Figure 5: The distributions of $P_{t} / E_{\text {beam }}$ after the cut on the jet charge for events in analysis (C) region II. Data and background contributions are shown in (a). The background sources are shaded as in Fig. 1. In (b) predictions from the simulation for chargino and neutralino events are shown: $m_{\tilde{\chi}_{1}^{+}}=90 \mathrm{GeV}$ and $m_{\tilde{\chi}_{1}^{0}}=70 \mathrm{GeV}$ (solid line), $m_{\tilde{\chi}_{1}^{+}}=$ $90 \mathrm{GeV}$ and $m_{\tilde{\chi}_{1}^{0}}=45 \mathrm{GeV}$ (dashed line) and $m_{\tilde{\chi}_{2}^{0}}=95 \mathrm{GeV}$ and $m_{\tilde{\chi}_{1}^{0}}=85 \mathrm{GeV}$ (dotted line). The normalisations of the signal distributions are arbitrary. The arrows shown indicate the selection criteria.

\section{OPAL}
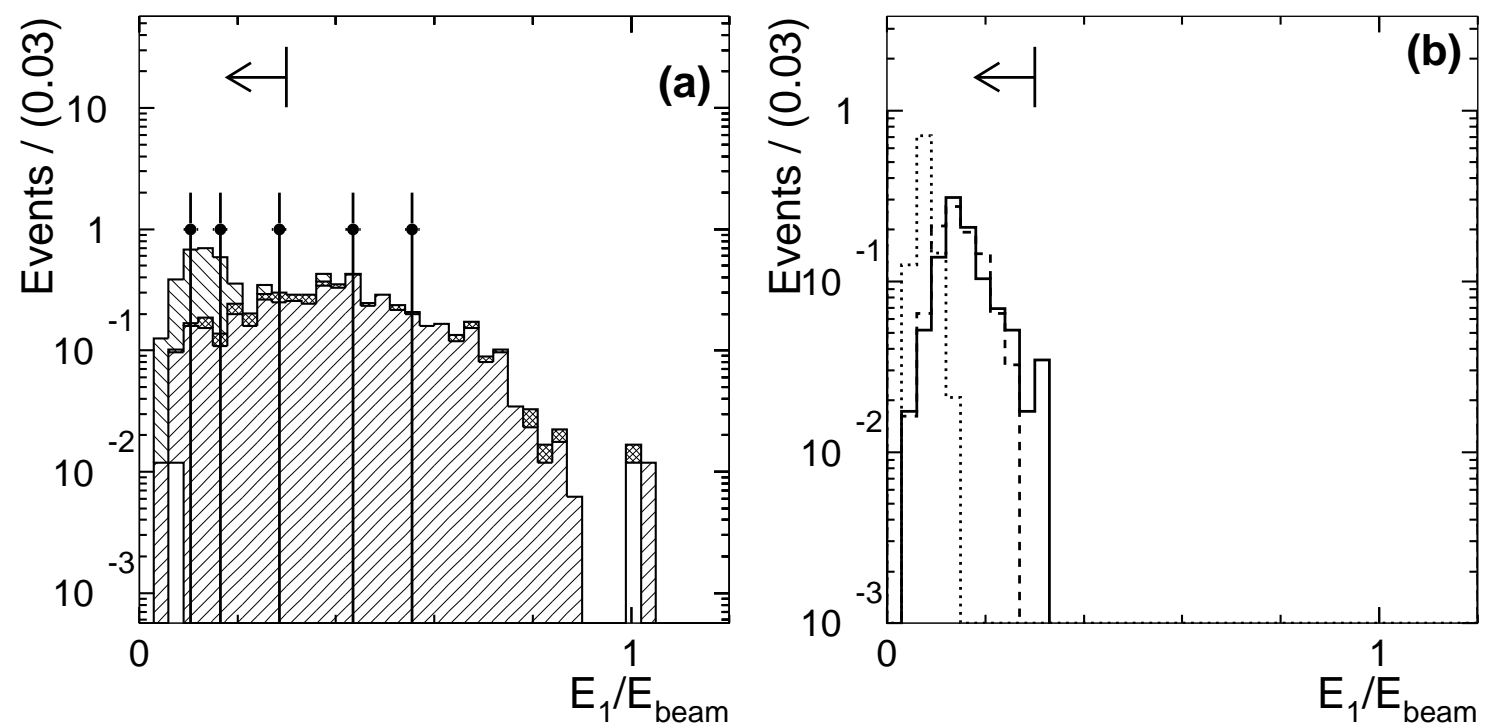

Figure 6: Distributions of the highest jet energy in analysis (C) region II after all other cuts have been applied. Data and background contributions are shown in (a). The background sources are shaded as in Fig. 1. The Monte Carlo signal distributions are shown in (b) and are labelled as in Fig. 5. The normalisations of the signal distributions are arbitrary. The arrows shown indicate the selection criteria. 


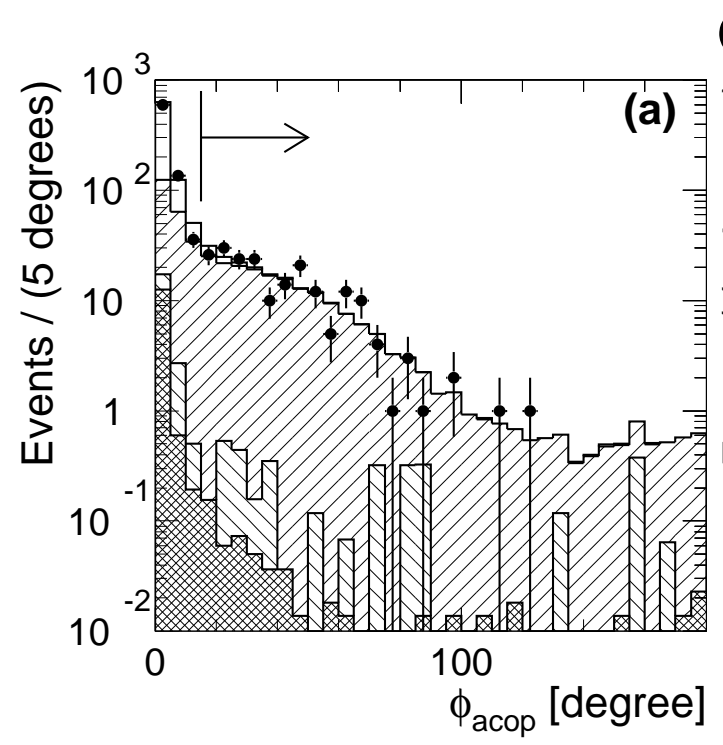

OPAL

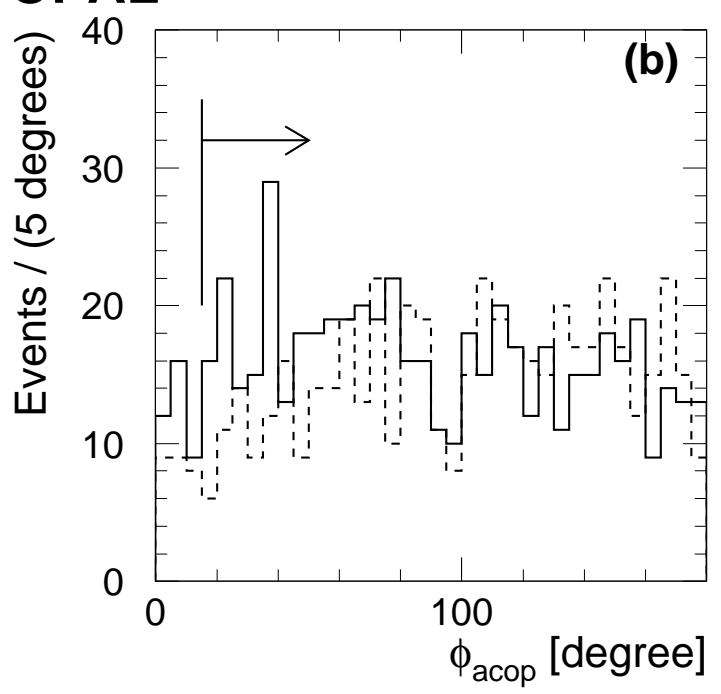

Figure 7: The distributions of acoplanarity angle in analysis (D) region ii after cut on $P_{t}$. Data and background contributions are shown in (a). The background sources are shaded as in Fig. 1. The Monte Carlo signal distributions are shown in (b) for $m_{\tilde{\chi}_{2}^{0}}=105 \mathrm{GeV}$ and $m_{\tilde{\chi}_{1}^{0}}=75 \mathrm{GeV}$ (solid line) and for $m_{\tilde{\chi}_{2}^{0}}=100 \mathrm{GeV}$ and $m_{\tilde{\chi}_{1}^{0}}=80 \mathrm{GeV}$ (dashed line). The normalisations of the signal distributions are arbitrary. The arrows shown indicate the selection criteria.

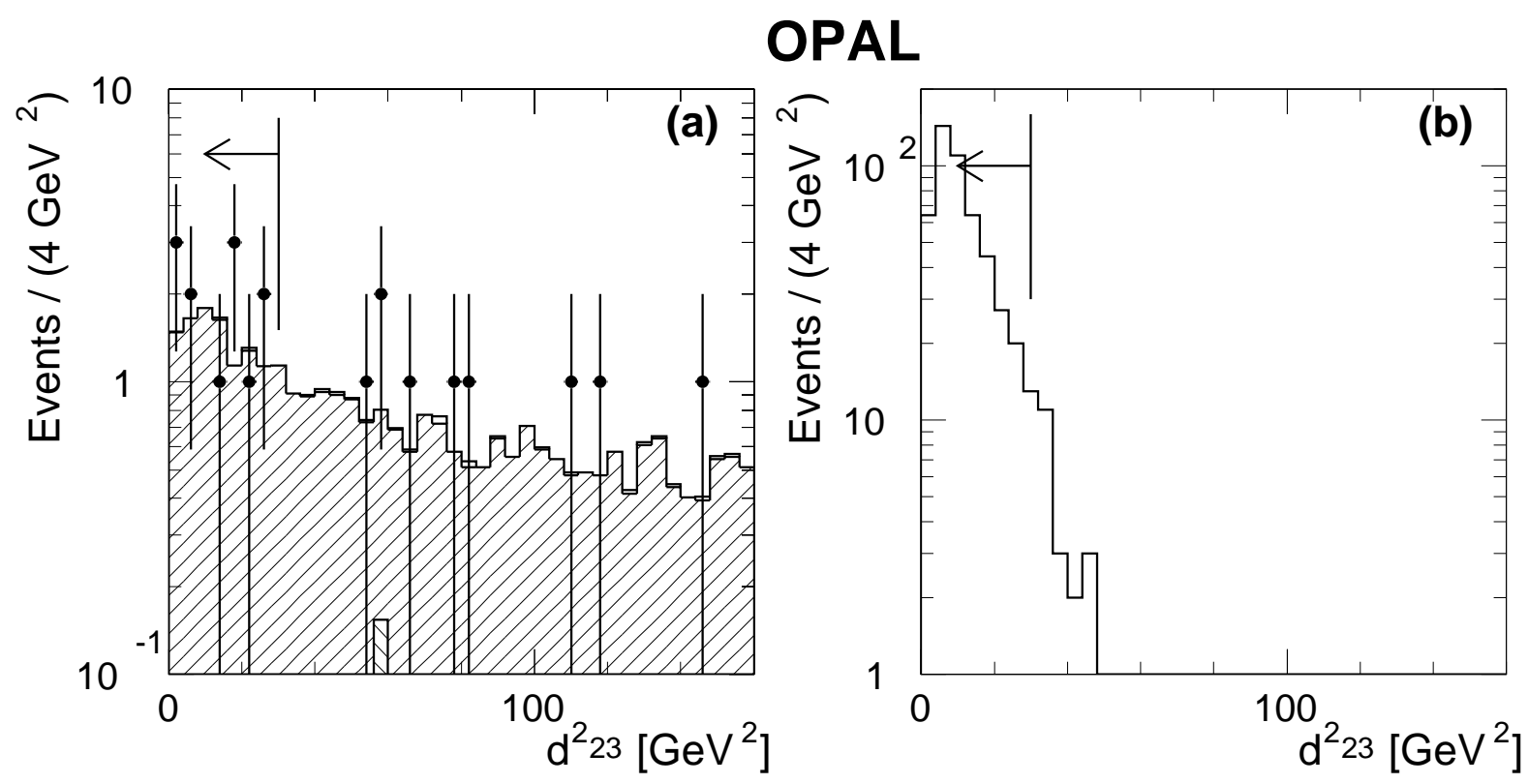

Figure 8: The distributions of $d_{23}\left(\equiv y_{23} E_{\text {vis }}^{2}\right) \mathrm{GeV}^{2}$ in analysis (D) region iv for three jet events after all the other cuts. Data and background contributions are shown in (a). The background sources are shaded as in Fig. 1. The Monte Carlo signal distribution is shown in (b) for $m_{\tilde{\chi}_{2}^{0}}=150 \mathrm{GeV}$ and $m_{\tilde{\chi}_{1}^{0}}=20 \mathrm{GeV}$. The normalisation of the signal distribution is arbitrary. The arrows shown indicate the selection criteria. 

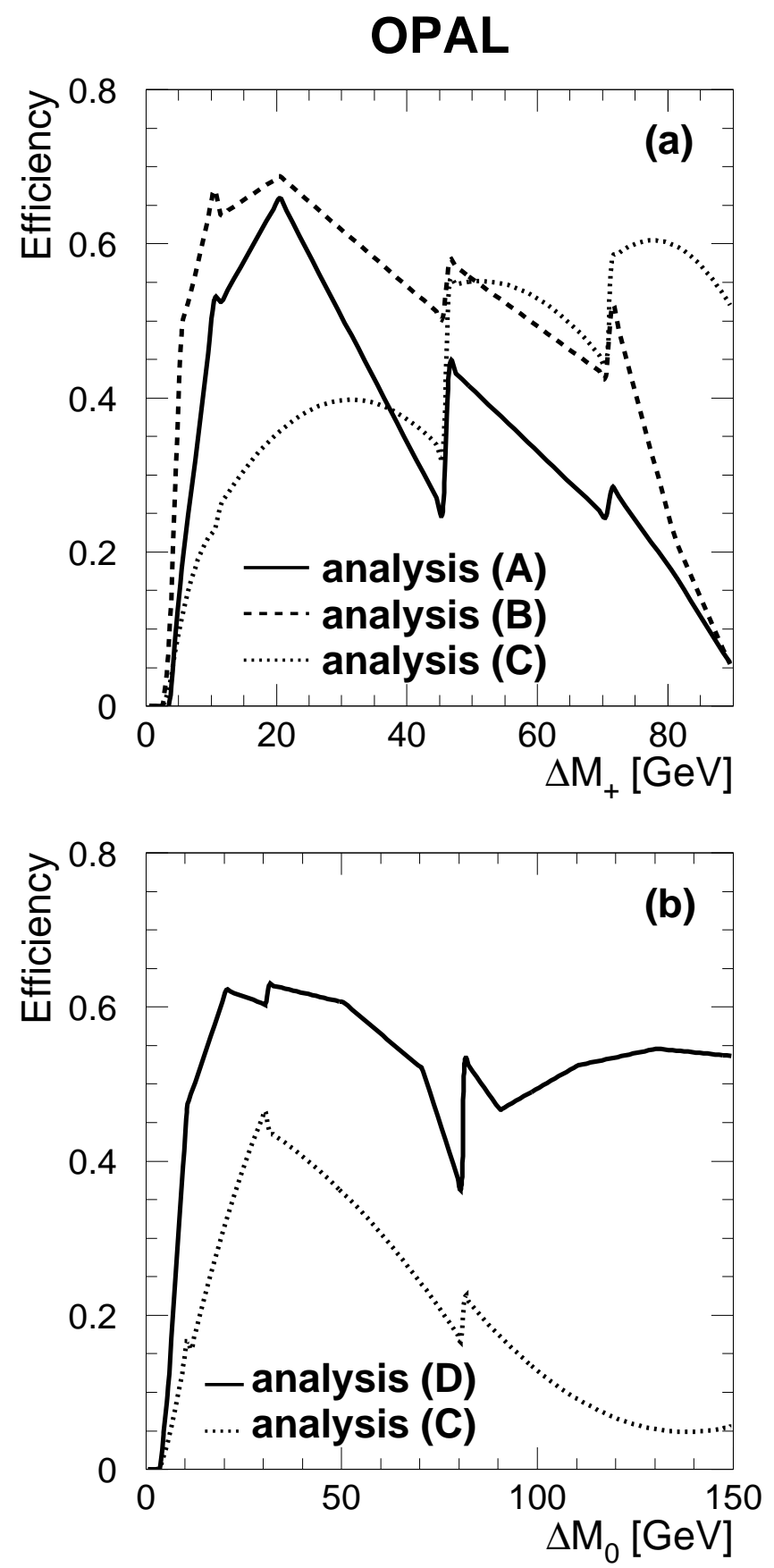

Figure 9: (a) Detection efficiencies of analyses (A) (solid line), (B) (dashed line) and (C) (dotted line) for $\tilde{\chi}_{1}^{+} \tilde{\chi}_{1}^{-}$events as a function of $\Delta M_{+} \equiv m_{\tilde{\chi}_{1}^{+}}-m_{\tilde{\chi}_{1}^{0}}$, for $m_{\tilde{\chi}_{1}^{+}}=90 \mathrm{GeV}$. (b) Detection efficiencies of analyses (C) (dotted line) and (D) (solid line) for $\tilde{\chi}_{1}^{0} \tilde{\chi}_{2}^{0}$ events as a function of $\Delta M_{0} \equiv m_{\tilde{\chi}_{2}^{0}}-m_{\tilde{\chi}_{1}^{0}}$, for $m_{\tilde{\chi}_{1}^{0}}+m_{\tilde{\chi}_{2}^{0}}=170 \mathrm{GeV}$. The efficiencies for each analysis are normalised to the number of events in each category. 


\section{OPAL}

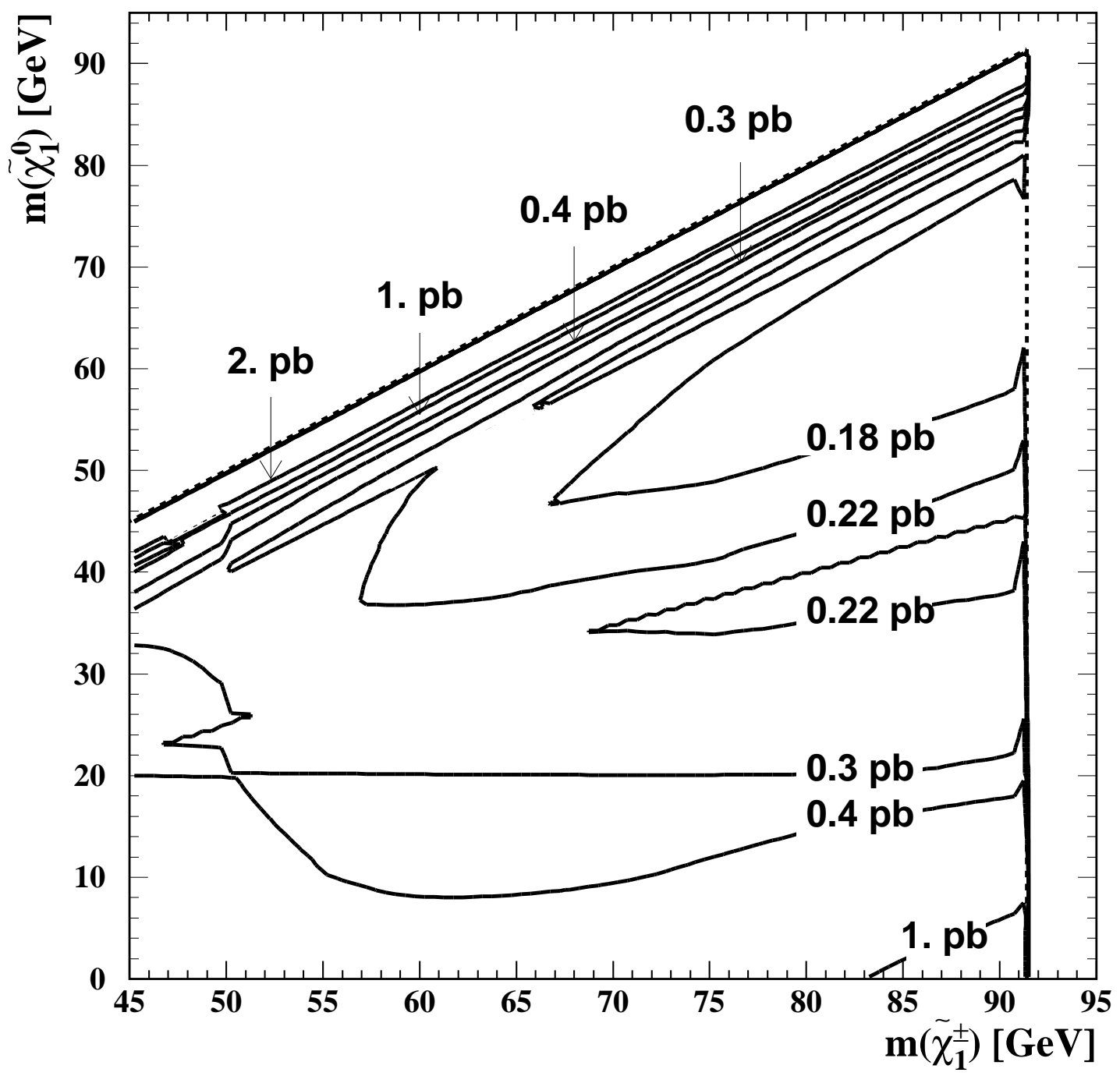

Figure 10: The contours of the $95 \%$ C.L. upper limits for the $\mathrm{e}^{+} \mathrm{e}^{-} \rightarrow \tilde{\chi}_{1}^{+} \tilde{\chi}_{1}^{-}$production cross-sections at $\sqrt{s}=182.7 \mathrm{GeV}$ are shown assuming $\operatorname{Br}\left(\tilde{\chi}_{1}^{+} \rightarrow \tilde{\chi}_{1}^{0} \mathrm{~W}^{(*)+}\right)=100 \%$. 


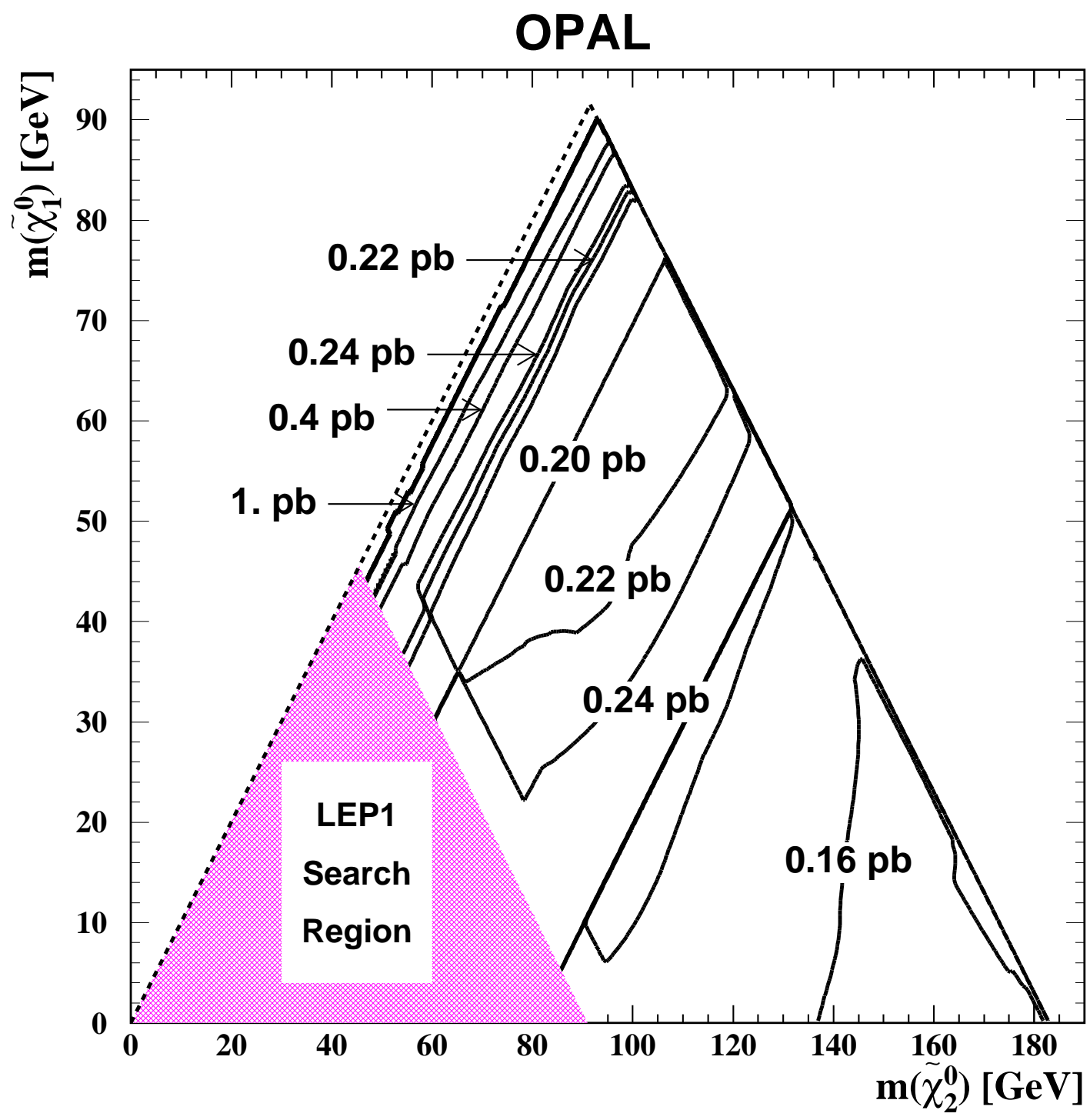

Figure 11: The contours of the 95\% C.L. upper limits for the $\mathrm{e}^{+} \mathrm{e}^{-} \rightarrow \tilde{\chi}_{2}^{0} \tilde{\chi}_{1}^{0}$ production cross-sections at $\sqrt{s}=182.7 \mathrm{GeV}$ are shown assuming $\operatorname{Br}\left(\tilde{\chi}_{2}^{0} \rightarrow \tilde{\chi}_{1}^{0} \mathrm{Z}^{(*)}\right)=100 \%$. The region for which $m_{\tilde{\chi}_{2}^{0}}+m_{\tilde{\chi}_{1}^{0}}<m_{\mathrm{Z}}$ is not considered in this analysis. 


\section{OPAL}
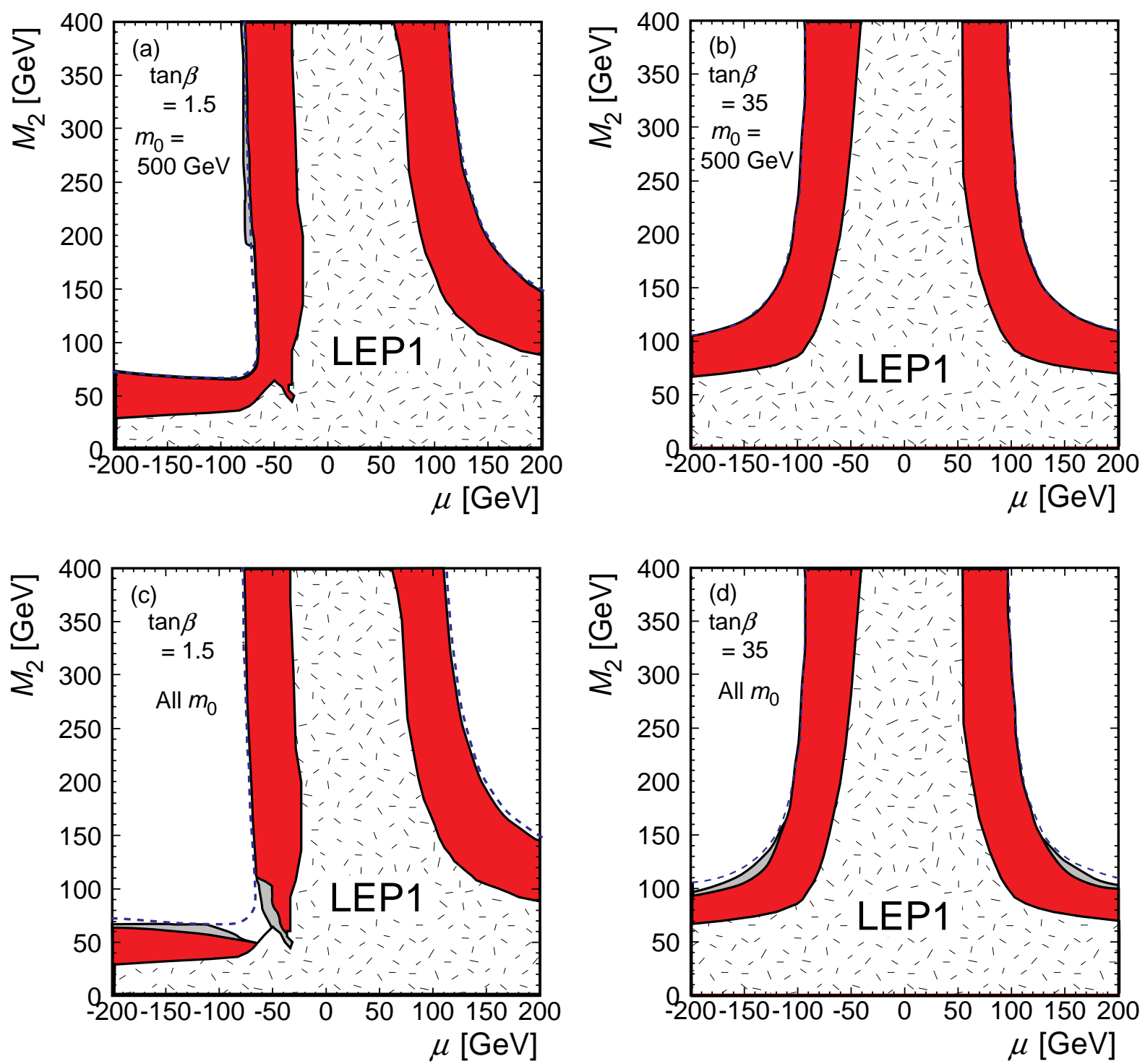

Figure 12: Exclusion regions at $95 \%$ C.L. in the $\left(M_{2}, \mu\right)$ plane with $m_{0} \geq 500 \mathrm{GeV}$ for (a) $\tan \beta=1.5$ and for (b) $\tan \beta=35$. Exclusion regions valid for all $m_{0}$ for (c) $\tan \beta=1.5$ and for $(\mathrm{d}) \tan \beta=35$. The speckled areas show the LEP1 excluded regions and the dark shaded areas show the additional exclusion region using the data from $\sqrt{s}=181$ $184 \mathrm{GeV}$. The kinematical boundaries for $\tilde{\chi}_{1}^{+} \tilde{\chi}_{1}^{-}$production are shown by the dashed curves. The light shaded region in (a) extending beyond the kinematical boundary of the $\tilde{\chi}_{1}^{+} \tilde{\chi}_{1}^{-}$production is obtained due to the interpretation of the results from the direct neutralino searches. The light shaded regions elsewhere show the additional exclusion regions due to direct neutralino searches and other OPAL search results (see text). 


\section{OPAL}
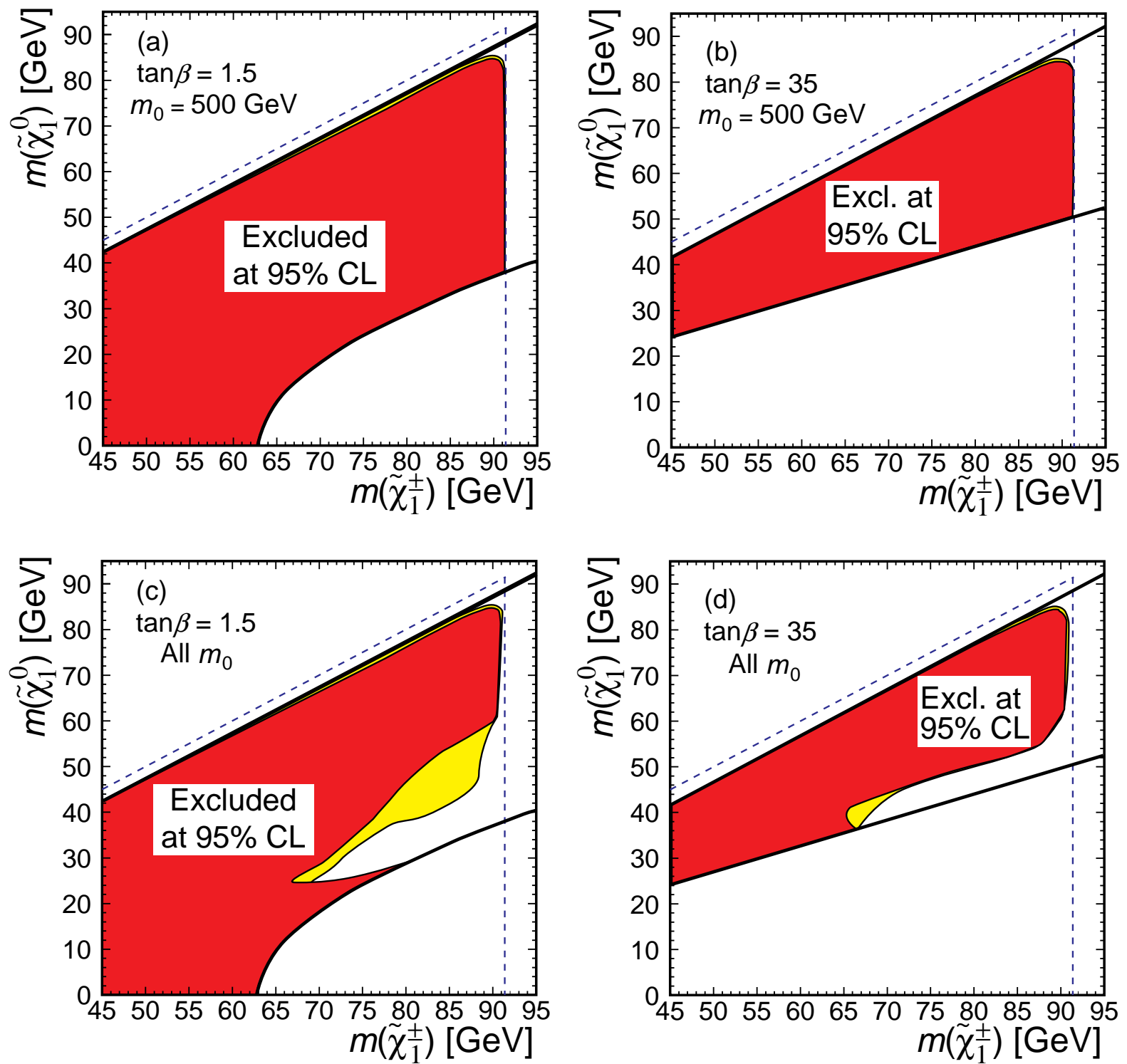

Figure 13: The $95 \%$ C.L. excluded region in the $\left(m_{\tilde{\chi}_{1}^{0}}, m_{\tilde{\chi}_{1}^{+}}\right)$plane within the framework of the CMSSM with $m_{0} \geq 500 \mathrm{GeV}$ for (a) $\tan \beta=1.5$ and for (b) $\tan \beta=35$. The excluded region valid for all $m_{0}$ for (c) $\tan \beta=1.5$ and for (d) $\tan \beta=35$. The thick solid lines represent the theoretical bounds of the CMSSM parameter space as given in the text. The kinematical boundaries for $\tilde{\chi}_{1}^{+} \tilde{\chi}_{1}^{-}$production and decay at $\sqrt{s}=182.7 \mathrm{GeV}$ are shown by dashed lines. The light shaded areas show the additional exclusion regions due to direct neutralino searches and other OPAL search results (see text). 


\section{OPAL}
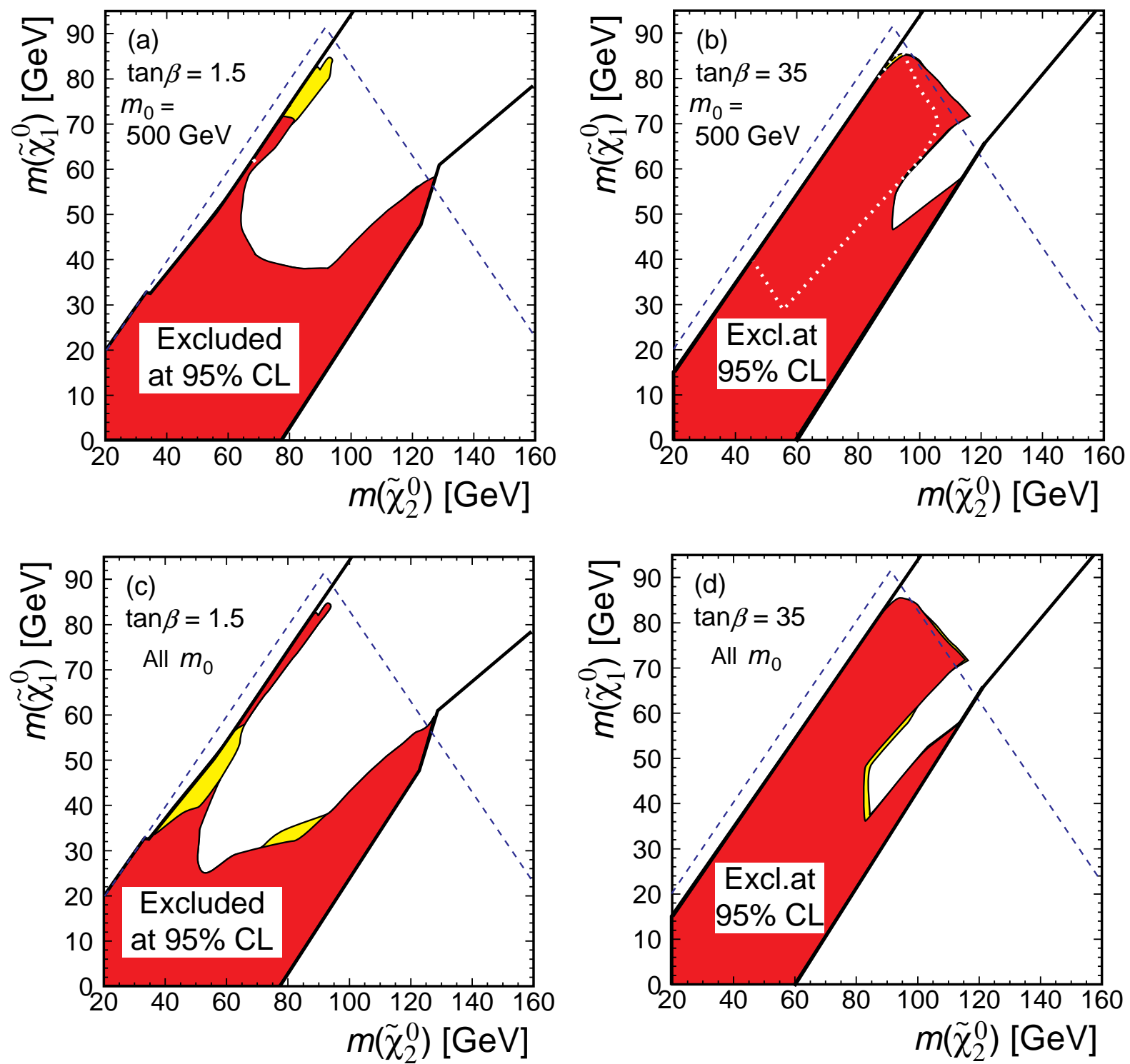

Figure 14: The 95\% C.L. excluded region in the $\left(m_{\tilde{\chi}_{1}^{0}}, m_{\tilde{\chi}_{2}^{0}}\right)$ plane within the framework of the CMSSM with $m_{0} \geq 500 \mathrm{GeV}$ for (a) $\tan \beta=1.5$ and for (b) $\tan \beta=35$. The excluded region valid for all $m_{0}$ for (c) $\tan \beta=1.5$ and for (d) $\tan \beta=35$. The thick solid lines represent the theoretical bounds of the CMSSM parameter space as defined in the text. The kinematical boundaries for $\tilde{\chi}_{2}^{0} \tilde{\chi}_{1}^{0}$ production and decay at $\sqrt{s}=183 \mathrm{GeV}$ are shown by dashed lines. The regions excluded outside of the kinematical boundary $m_{\tilde{\chi}_{2}^{0}}+m_{\tilde{\chi}_{1}^{0}}=\sqrt{s}$ is due to the interpretation of the $\tilde{\chi}_{1}^{+} \tilde{\chi}_{1}^{-}$search results. The dark region is excluded by the results of direct $\tilde{\chi}_{1}^{+} \tilde{\chi}_{1}^{-}$searches. The light shaded areas show the additional exclusion regions due to direct neutralino searches and other OPAL search results (see text). The regions inside the white dotted lines in Fig.(a) and (b) would be excluded by the direct neutralino searches alone. 


\section{OPAL}
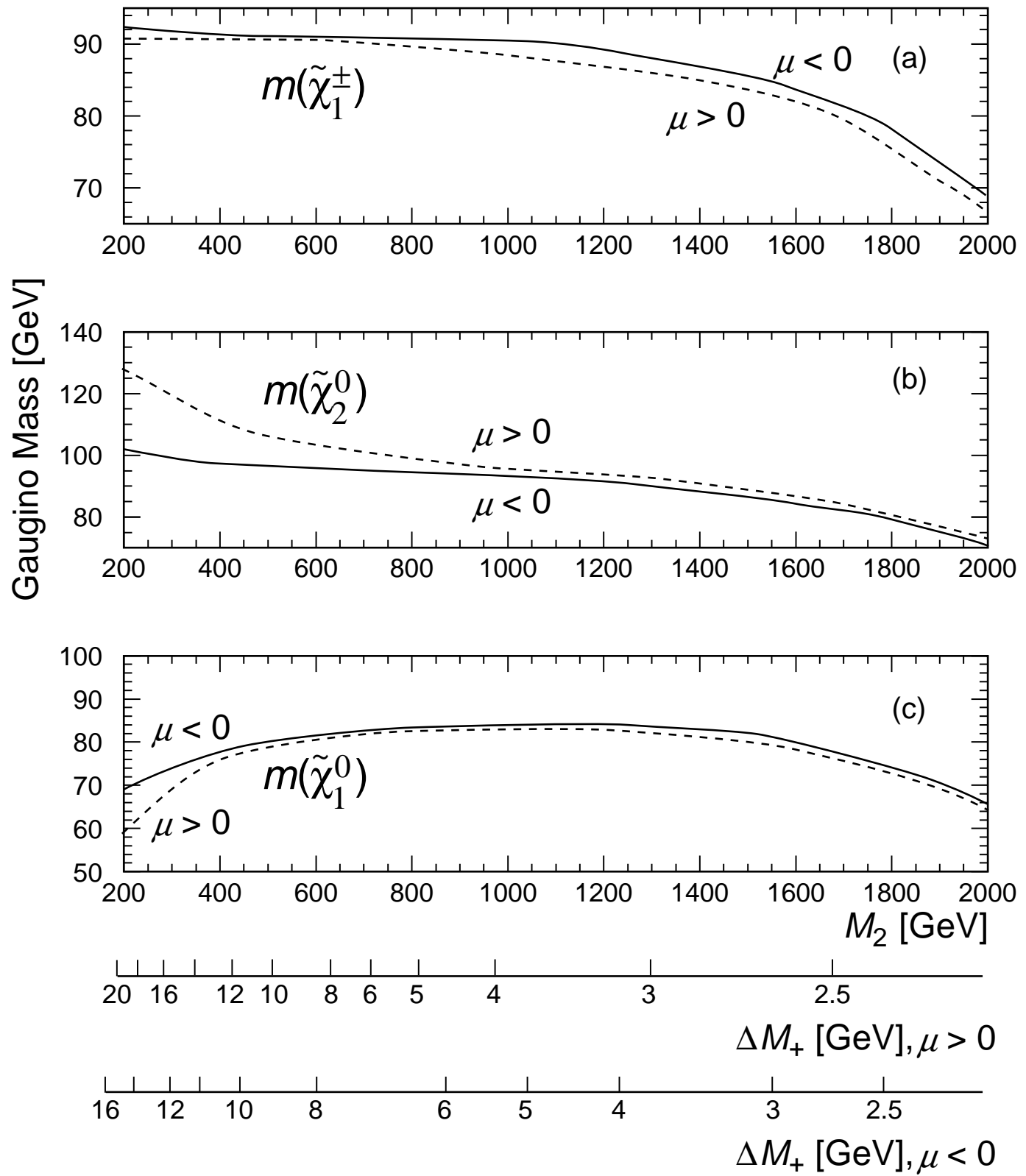

Figure 15: The $95 \%$ C.L. lower mass limits for (a) $\tilde{\chi}_{1}^{+}$, (b) $\tilde{\chi}_{2}^{0}$, and (c) $\tilde{\chi}_{1}^{0}$ for $\tan \beta=1.5$ and $m_{0}=500 \mathrm{GeV}$ for slices of constant value of $M_{2}$. Limits are shown separately for $\mu<0$ (solid lines) and $\mu>0$ (dashed lines). Curves for larger values of $\tan \beta$ are in general between those shown for $\mu<0$ and $\mu>0$. The corresponding values of mass difference $\Delta M_{+}$between the chargino and the lightest neutralino are also shown on scales below the plots. 


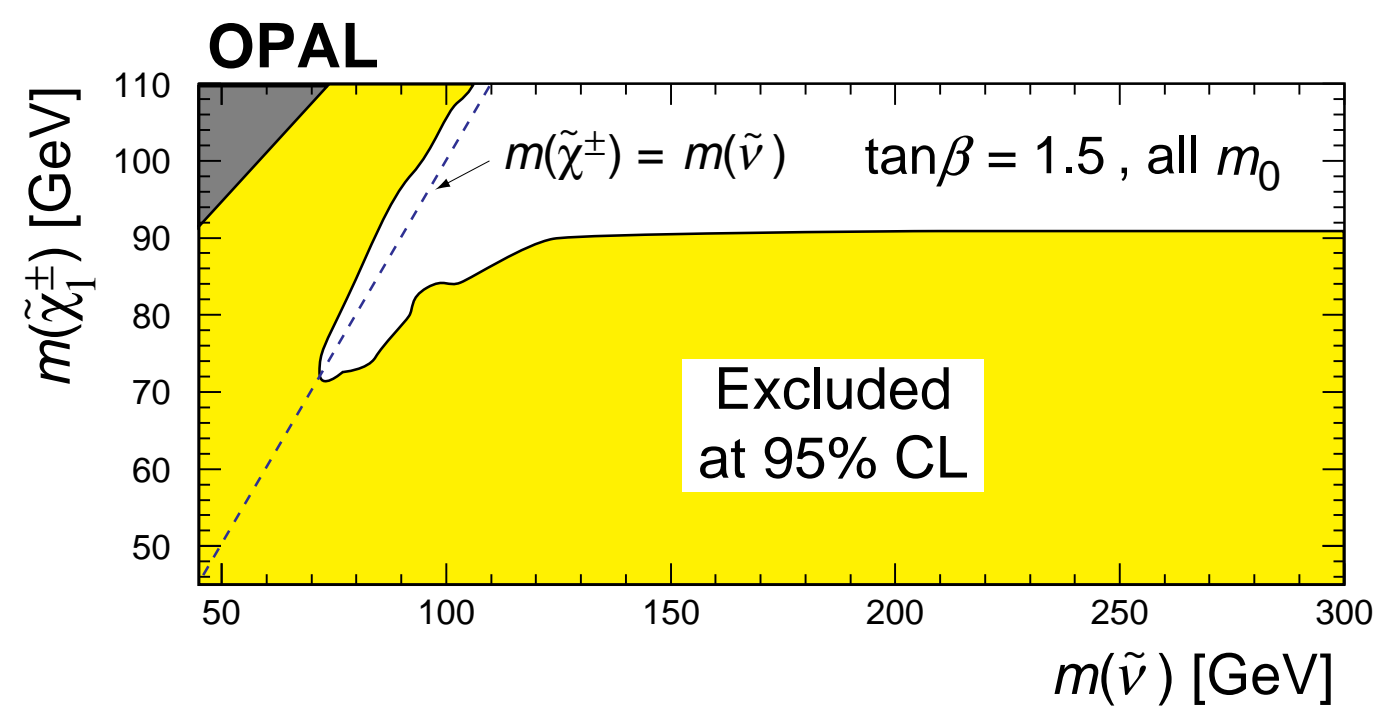

Figure 16: The $95 \%$ C.L. excluded region in the $\left(m_{\tilde{\nu}}, m_{\tilde{\chi}_{1}^{+}}\right)$plane for $\tan \beta=1.5$ and for all $m_{0}$ values. The region close to the line of $m_{\tilde{\nu}}=m_{\tilde{\chi}^{+}}$where the $\tilde{\chi}_{1}^{+} \tilde{\chi}_{1}^{-}$search fails due to small visible energy is excluded using the limits for sneutrinos and OPAL limits for sleptons. The dark shaded region is theoretically inaccessible. 


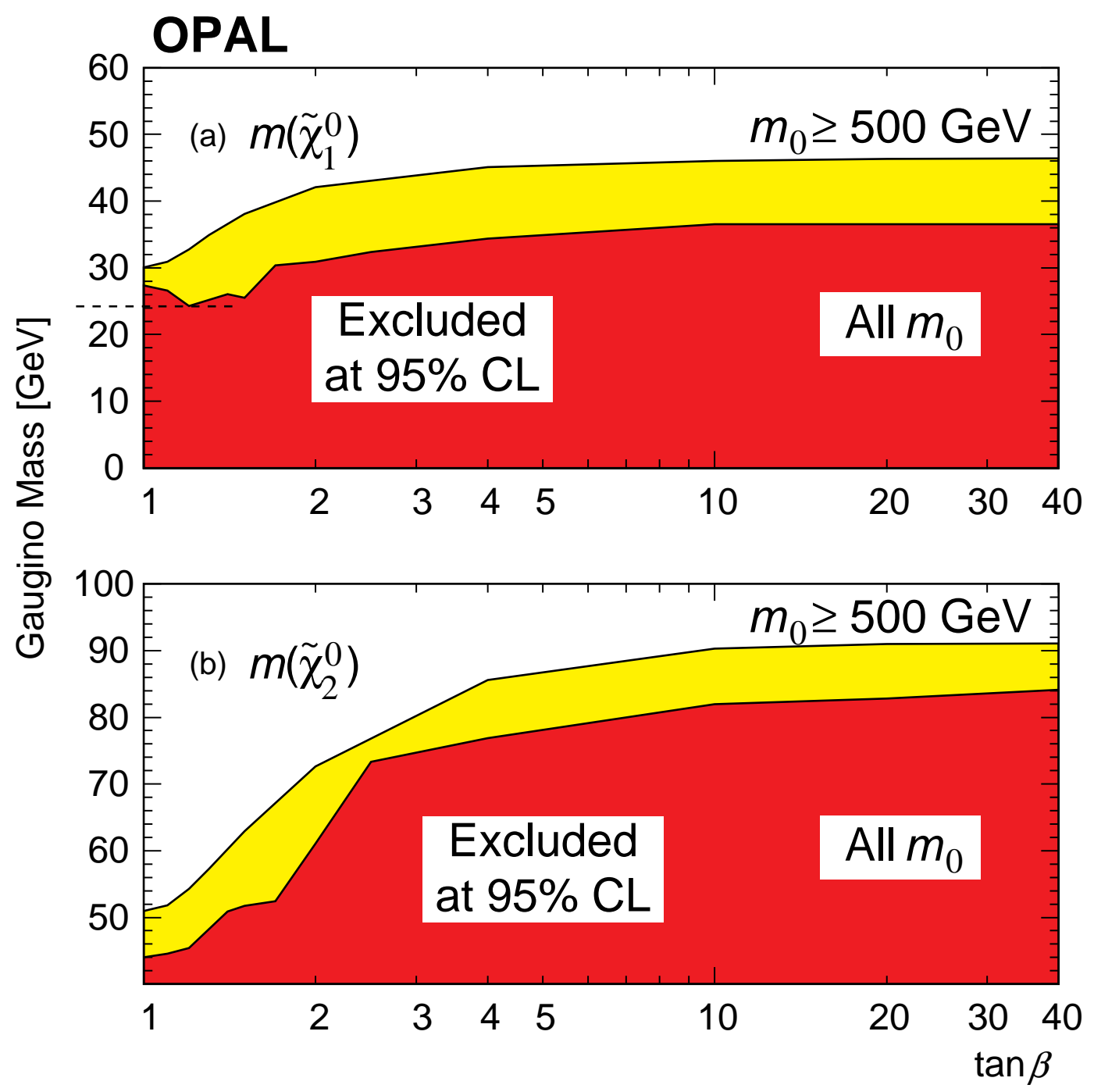

Figure 17: The 95\% C.L. mass limit on (a) the lightest neutralino $\tilde{\chi}_{1}^{0}$ and (b) the secondlightest neutralino $\tilde{\chi}_{2}^{0}$ as a function of $\tan \beta$ for $m_{0} \geq 500 \mathrm{GeV}$. The mass limit on $\tilde{\chi}_{2}^{0}$ is for the additional requirement of $\Delta M_{0}>10 \mathrm{GeV}$. The exclusion region for $m_{0} \geq 500 \mathrm{GeV}$ is shown by the light shaded area and the excluded region valid for all $m_{0}$ values by the dark shaded area. 